\title{
Predicting Complex Non-spherical Instability Shapes of Inertial Cavitation Bubbles in Viscoelastic Soft Matter
}

\author{
Jin Yang, ${ }^{1}$ Anastasia Tzoumaka, ${ }^{2}$ Kazuya Murakami, ${ }^{3}$ Eric Johnsen, ${ }^{3}$ David L. Henann, ${ }^{2}$ and Christian Franck ${ }^{1, *}$ \\ ${ }^{1}$ Department of Mechanical Engineering, University of Wisconsin-Madison \\ ${ }^{2}$ School of Engineering, Brown University \\ ${ }^{3}$ Department of Mechanical Engineering, University of Michigan, Ann Arbor
}

(Dated: April 8, 2021)

\begin{abstract}
Inertial cavitation in soft matter is an important phenomenon featured in a wide array of biological and engineering processes. Recent advances in experimental, theoretical, and numerical techniques have provided access into a world full of nonlinear physics, yet most of our quantitative understanding to date has been centered on a spherically symmetric description of the cavitation process. However, cavitation bubble growth and collapse rarely occur in a perfectly symmetrical fashion, particularly in soft materials. Predicting the onset of dynamically arising, non-spherical instabilities has remained a significant, unresolved challenge in part due to the additional constitutive complexities introduced by the surrounding nonlinear viscoelastic solid. Here, we provide a new theoretical model capable of accurately predicting the onset of non-spherical instability shapes of a bubble in a soft material by explicitly accounting for all pertinent nonlinear interactions between the fluid-like cavitation bubble and the solid-like surroundings. Comparison against high-resolution experimental images from laser-induced cavitation events in a polyacrylamide (PA) hydrogel show excellent agreement. Interestingly, and consistent with experimental findings, our model predicts the emergence of various dynamic instability shapes for hoop stretch ratios greater than one in contrast to most quasistatic investigations. Our new theoretical framework not only provides unprecedented insight into the cavitation dynamics in a soft solid, but it also provides a quantitative means of interpreting bubble dynamics relevant to a wide array of engineering and medical applications as well as natural phenomena.
\end{abstract}

Cavitation is the process whereby vapor cavities, or bubbles, are produced in a liquid or solid due to pressure differences. For inertially generated bubbles, the resulting dynamics are highly transient and often nonlinear. While models have been developed to successfully describe cavitation dynamics in water, the extension of the theoretical foundation of these models to soft materials is challenging due to their unique deformation and failure mechanisms, especially under extreme loading conditions (large strain magnitudes and high strain rates). In particular, the nature of the physical and chemical composition of many soft polymers gives rise to highly nonlinear elastic and viscoelastic macroscale material behavior.

Current prediction of inertial cavitation dynamics usually relies on the approximation that the cavitation process remains nominally spherically symmetric through its life cycle, allowing for the use of the classical RayleighPlesset [1-5] or Keller-Miksis [6-8] modeling approaches. However, recent experiments and numerical simulations show that cavitation bubble growth and collapse rarely occur in a perfectly spherically symmetric fashion even in nominally homogeneous and isotropic soft matter [9-13]. Predicting the onset of non-spherical deformation arising due to instabilities during inertial cavitation in soft viscoelastic materials is an important and fundamental problem with significant implications across many applications. For example, non-spherical instabilities may lead to strain-localization and subsequent material damage near the bubble wall as well as other important physical, chemical, and biological outcomes, including sono- chemistry [14, 15], sonoluminesence [9], local plasticity and fracture, and/or tissue/cell dysfunctions in biological materials [16].

Documentation of cavitation-related instabilities in liquids has a well-established history in the fluid mechanics community, including detailed descriptions of classical instability phenomena, such as the Rayleigh-Taylor (RT) instability [17], or parametric instabilities, which arise due to the accumulation of non-spherical perturbations over many oscillation periods $[10,13,18,19]$. However, predicting the onset of non-spherical instabilities during inertial cavitation in soft materials is still in its infancy, largely due to the additional complexities arising from the intrinsic coupling of the bubble dynamics to a nonlinear, viscoelastic solid. Cavitation in a solid material can present different and perhaps more complex (non-spherical) instability patterns compared to a fluid, including wrinkles, creases, and folds. In addition, parametric instabilities, tend to occur earlier during the cavitation expansion-collapse cycles in a solid material when compared to a fluid. Mathematically, the constitutive laws of hyperelastic solids are typically expressed using a Lagrangian description based on the reference configuration, while the cavitation dynamics are typically described in the current, deformed configuration, which must be accounted for when theoretically describing and predicting the onset and evolution of inertial cavitation instability patterns within a soft solid.

To address these challenges, we present a new theoretical framework that, based on a first-order incremental 

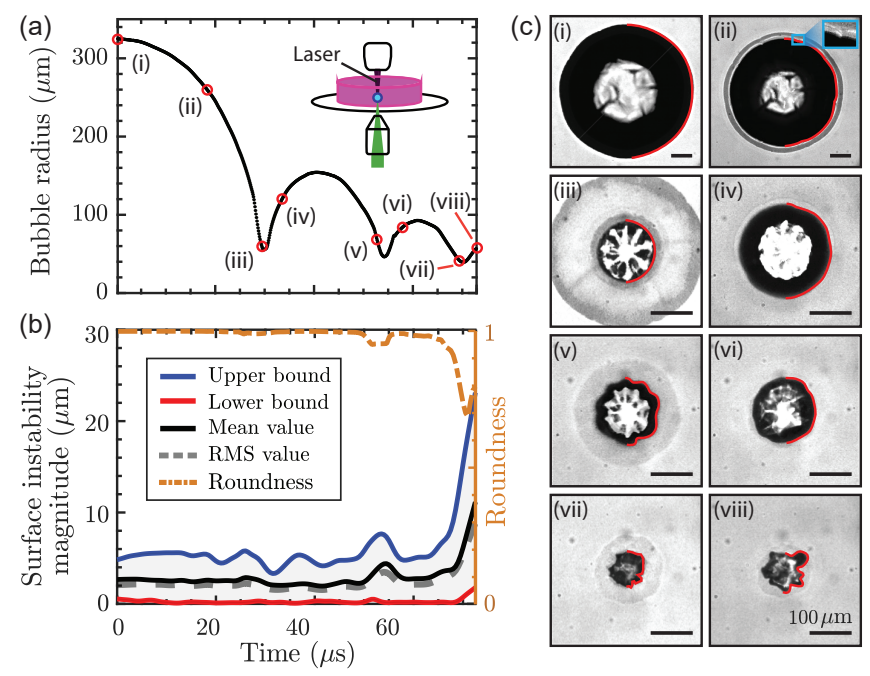

FIG. 1. Spatiotemporally recorded bubble dynamics via highspeed videography [8]. (a) A representative bubble radius vs. time curve for a laser-induced cavitation bubble inside a PA gel. (b) Quantitative measurements of the magnitude of bubble surface instabilities. (c) Various types of non-spherical bubble shapes corresponding to different time-points (red circles) in (a). Red dashed lines are overlaid onto original images to visualize the shape of the bubble wall. Inset in (c-ii) is the zoomed-in non-spherical shape instability. Data from [8].

perturbation analysis, is able to accurately capture and predict the evolution of complex deformation modes observed in dynamic cavitation events. By comparing our theoretical predictions against recent experimental observations of various non-spherical bubble shapes, we find that our model is in excellent quantitative and qualitative agreement with the experimental measurements.

In order to compare and assess the accuracy of our theoretical predictions against appropriate experiments, we take advantage of several existing high-resolution data sets of laser-induced cavitation in polyacrylamide (PA) hydrogels [8]. A representative bubble radius vs. time curve is shown in Fig. 1(a), plotted starting at the time-point of the maximum bubble radius $R_{\max }$. Figure 1(c) depicts various non-spherical bubble shapes at eight time-points during the expansion and collapse cycle of the cavitation dynamics, as indicated by red circles in Fig. 1(a). The bubble shapes in frames (i,ii,iv,vi) show nearly spherical bubbles, while frames (v,vii,viii) present wrinkling, creasing, and stellate instabilities near the bubble wall, respectively. From these experimental observations, we quantify the upper and lower bounds, mean, and root-mean-square (RMS) values of the magnitude of these bubble surface instabilities [20], as well as the bubble roundness $\left(4 \pi \times\right.$ Area Perimeter $\left.^{2}\right)$ (see Fig. 1(b)).

Next, we non-dimensionalize all bubble radius vs. time curves $\left(t^{*}=t \sqrt{p_{\infty} / \rho} / R_{\max }, R^{*}=R / R_{\max }\right.$, where $p_{\infty}$

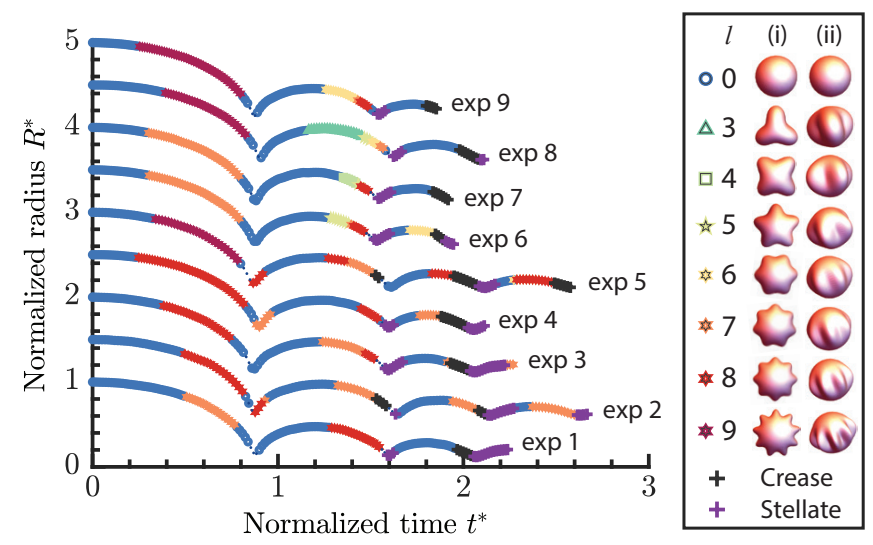

FIG. 2. Summary of various instability patterns in nine individual cavitation events. Experimental data sets 2-9 are purposefully offset vertically for easier viewing of each curve. Right inset: (i) Top and (ii) side views of different instability modes.

and $\rho$ are defined after Eq. (1)), and present our findings for the various instability patterns for nine individual experiments in Fig. 2. Experimental data sets 2-9 are purposefully offset vertically for easier viewing of each curve. Top and side views of different instability modes (mode shape number $l$ later defined in Eq. (4)) are summarized in the right inset. The instability pattern within each frame is directly extracted from that frame. Creases are distinguishable from wrinkles by their innate surface folds and significant local curvature in the formed creases (Fig. 1(c-vii)) [21], whereas the emergence of stellate patterns are typically observed right after the occurrence of local creases (Fig. 1(c-viii)). By conducting a careful qualitative shape analysis, we find the first appearance of non-spherical bubble shapes near the first, often violent (local Mach number exceeding 0.08), collapse, predominantly featuring shapes similar to the spherical harmonic function of mode shape 8 (Fig. 1(c-v)), while subsequent collapse points show the emergence of other mode shapes (e.g., spherical harmonic function mode shapes 6 and 7 ) along with mode shape 8 .

To quantify the critical condition for the onset of non-spherical instabilities associated with each mode shape, we develop a new theoretical framework based on a perturbation approach of the spherically symmetric Rayleigh-Plesset governing equation [22], in which the time-dependent bubble radius of the base state $R(t)$ is governed by $[7,8,18,22]$ :

$$
R R_{, t t}+\frac{3}{2} R_{, t}^{2}=\frac{1}{\rho}\left(p_{b}-p_{\infty}+S-\frac{2 \gamma}{R}\right),
$$

where $(\cdot)_{, t}$ denotes the derivative with respect to time; $\rho$ is the mass density of the surrounding viscoelastic material, which is assumed to be nearly constant; $\gamma$ is the surface tension between the gaseous bubble phase and the 
surrounding medium; $p_{b}$ is the internal bubble pressure; $p_{\infty}$ is the far-field pressure, which is assumed to be atmospheric as there are no external driving forces; and $S$ is given through the integral of deviatoric Cauchy stress components over the surroundings. Consistent with our previous work, we describe the surrounding soft material as a nonlinear strain-stiffening Kelvin-Voigt (qKV) viscoelastic material [8] with a quadratic-law strain energy density function $W$ given by

$$
W=\frac{G}{2}\left[\left(I_{1}-3\right)+\frac{\alpha}{2}\left(I_{1}-3\right)^{2}\right],
$$

where $I_{1}$ is the first invariant of the right Cauchy-Green deformation tensor, $G$ is the ground-state shear modulus, and $\alpha$ is a dimensionless material parameter characterizing large deformation strain stiffening effects [8]. The Newtonian viscosity $\mu$ in the qKV model is assumed to be constant and typically has a value of $O\left(10^{-3}\right) \sim O\left(10^{-1}\right)$ $\mathrm{Pa} \cdot \mathrm{s}$ for water-based hydrogels and biomaterials [8, 23]. The stress integral $S$ then takes the form

$$
\begin{aligned}
S= & \frac{(3 \alpha-1) G}{2}\left[5-\left(\frac{R_{0}}{R}\right)^{4}-\frac{4 R_{0}}{R}\right]-\frac{4 \mu \dot{R}}{R}+ \\
& 2 \alpha G\left[\frac{27}{40}+\frac{1}{8}\left(\frac{R_{0}}{R}\right)^{8}+\frac{1}{5}\left(\frac{R_{0}}{R}\right)^{5}+\left(\frac{R_{0}}{R}\right)^{2}-\frac{2 R}{R_{0}}\right]
\end{aligned}
$$

where $R_{0}$ is the undeformed bubble radius. We model the inside of the bubble as a two-phase mixture consisting of water vapor and non-condensible gas, which is homobaric and follows the ideal gas law. We neglect temperature changes at the bubble wall and assume there is no mass diffusion of non-condensible gas across the bubble wall since both the heat and mass diffusion processes across the bubble wall are much slower than the bubble dynamics. Simulations are initiated when the bubble attains its maximum radius $R_{\max }$ thus avoiding the need to account for non-equilibrium nucleation and growth dynamics and beginning the simulations at thermodynamic equilibrium $[7,24]$.

Next, based on the spherically symmetric RayleighPlesset governing equation (1), we consider the following perturbation of the displacement field using spherical harmonic basis functions [25]:

$$
\left\{\begin{array}{l}
\tilde{u}_{r}=\frac{a(t) R^{2}}{r^{2}} Y_{l}^{m}(\theta, \phi) \quad(l \geqslant|m|>0) \\
\tilde{u}_{\theta}=0 \\
\tilde{u}_{\phi}=0
\end{array}\right.
$$

where $\{r, \theta, \phi\}$ are radial, polar, and azimuthal angular coordinates; $Y_{l}^{m}(\theta, \phi)$ is the normalized $(l, m)^{t h}$ spherical harmonic function; and $a(t)$ is the time-dependent perturbation magnitude at the bubble wall. One can show that the non-spherical perturbation (4) is isochoric [19].

The perturbation is governed by the momentum balance equation, expressed in the current, deformed con- figuration as

$$
\nabla \cdot \sigma=\rho \mathbf{a}
$$

where $\nabla \cdot(\bullet)$ is the spatial divergence operator, $\mathbf{a}$ is the acceleration vector, and $\boldsymbol{\sigma}$ is the Cauchy stress tensor. The traction boundary condition at the bubble wall is

$$
\left.\boldsymbol{\sigma} \mathbf{n}\right|_{r=R+a Y_{l}^{m}}=-p_{\mathrm{b}} \mathbf{n}-\gamma\left(\nabla_{\mathcal{S}} \cdot \mathbf{n}\right) \mathbf{n},
$$

where $[\mathbf{n}]=\left[-1, a Y_{l, \theta}^{m} / R, a Y_{l, \phi}^{m} /(R \sin \theta)\right]^{\top}$ is the linearized outward unit normal vector on the perturbed bubble wall, and $\nabla_{\mathcal{S}} \cdot(\bullet)$ is the surface divergence operator in the deformed configuration. In the far-field, the stress approaches a state of hydrostatic pressure: $\left.\boldsymbol{\sigma}\right|_{r \rightarrow \infty}=-p_{\infty} \mathbf{I}$, where $\mathbf{I}$ is the identity tensor.

After inserting (4) into (5), integrating over $r$ from the current, perturbed bubble wall to the far-field, applying the radial boundary conditions, and collecting the $O(a)$ terms, we obtain the governing, second-order differential equation for the bubble instability perturbation magnitude, $a$ :

$$
a_{, t t}+\eta a_{, t}-\xi a=0 ; \quad \eta=\frac{3 R_{, t}}{R}+\frac{4 \mu}{\rho R^{2}}+\frac{l(l+1) \mu}{3 \rho R^{2}},
$$

where $\xi$ explicitly accounts for inertial effects during cavitation, nonlinear deformations of the viscoelastic solid, and surface tension effects:

$$
\begin{aligned}
\xi= & -\frac{R_{, t t}}{R}+\frac{4 \mu R_{, t}}{\rho R^{3}}-\frac{2 l(l+1) \mu R_{, t}}{3 \rho R^{3}} \\
& -\frac{2 G R_{0}}{\rho R^{3}}\left(1+\frac{R_{0}^{3}}{R^{3}}\right)-\frac{G l(l+1)}{\rho\left(R^{2}+R R_{0}+R_{0}^{2}\right)} \\
& -\frac{2 \alpha G}{\rho R^{2}} \frac{\left(R-R_{0}\right)^{2}}{R R_{0}}\left(1+\frac{R_{0}}{R}\right)^{3}\left(2-\frac{2 R_{0}}{R}+\frac{3 R_{0}^{2}}{R^{2}}-\frac{R_{0}^{3}}{R^{3}}+\frac{R_{0}^{4}}{R^{4}}\right) \\
& -\frac{\alpha G l(l+1)\left(R-R_{0}\right)^{2}}{5 \rho R R_{0}\left(R^{2}+R R_{0}+R_{0}^{2}\right)}\left(10+\frac{6 R_{0}}{R}+\frac{3 R_{0}^{2}}{R^{2}}+\frac{R_{0}^{3}}{R^{3}}\right) \\
& -\frac{(l+2)(l-1) \gamma}{\rho R^{3}} .
\end{aligned}
$$

We note that when $\alpha \rightarrow 0$, Eqs. (7-8) describe the evolution of the perturbation magnitude $a$ in a neo-Hookean viscoelastic medium. Examining the differential relation (7), we find that perturbations $a$ grow if $\eta<0$ or $\xi>0$ and decay if $\eta>0$ and $\xi<0$ [26].

Utilizing the radius vs. time data from the experimental observations in Fig. 2 in our instability model Eqs. (78 ), we can theoretically predict the onset of various nonspherical instability patterns during the cavitation process. For illustration, based on this data set (specifically, "exp 4" in Fig. 2), we consider the emergence of instability mode shape $l=8$. The evolution of the bubble radius $R$ for this case is numerically simulated, and the histories of the bubble radius and the quantities $\eta$ and $\xi$ are plotted in Fig. 3(b-d). Conditions under which non-spherical instabilities corresponding to $l=8$ are predicted (i.e., $\eta<0$ or $\xi>0$ ) are marked as red shaded 

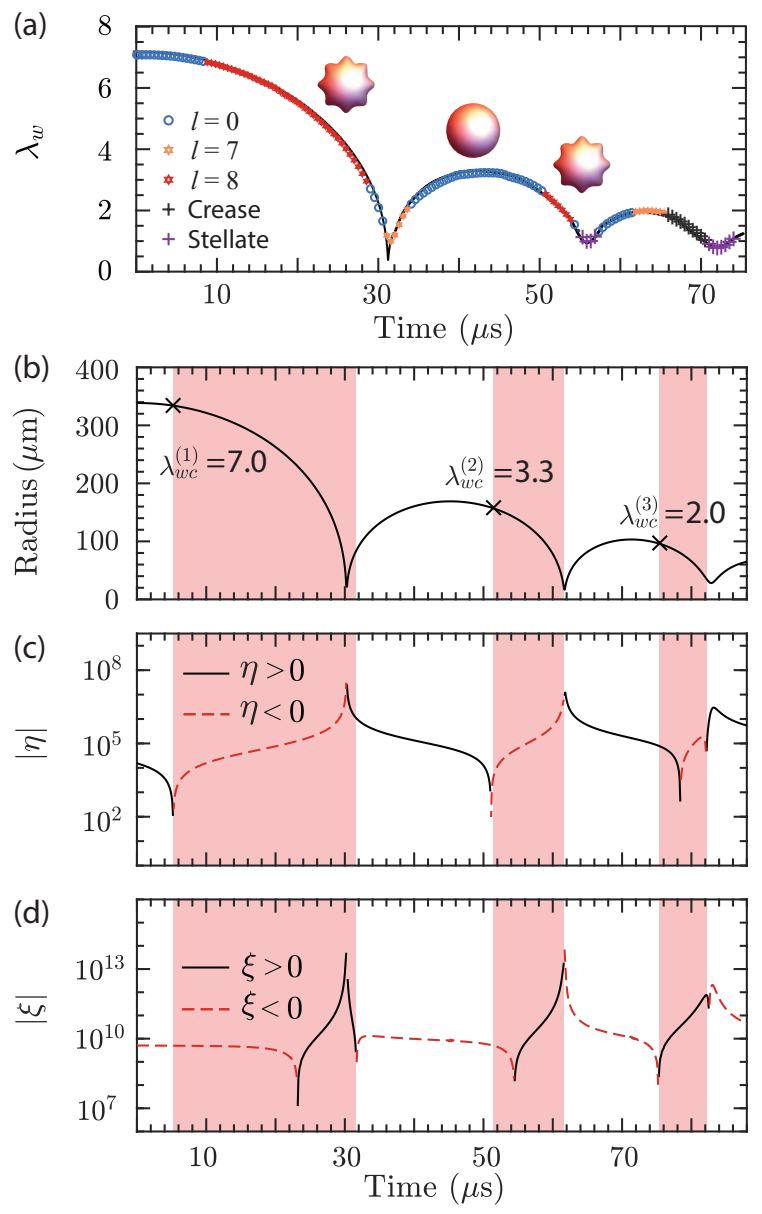

FIG. 3. (a) Hoop stretch at the bubble wall $\lambda_{w}$ for the experiment labeled "exp 4" in Fig. 2. Histories of (b) the bubble radius $R$ and the quantities (c) $\eta$ and (d) $\xi$ are numerically computed using simulation parameters based on Fig. 2 "exp 4". Red shaded regions denote situations in which non-spherical instabilities corresponding to $l=8$ are predicted. The critical values of $\lambda_{w}$ at the onset of instability occurring during the first three collapse cycles are marked as black crosses in (b).

regions in Fig. 3(b-d). The onset of non-spherical deformation predicted by the theoretical instability model is in good agreement with the experimental observations in Fig. 3(a) during each of the first three collapse cycles.

From Eqs. (7-8), we find that both material viscosity and surface tension always act to stabilize the bubble against non-spherical deformation. However, when the bubble size is much greater than the characteristic length $\gamma / G$, the effect of surface tension is negligible. When the bubble approaches the final equilibrium radius $\left(R \rightarrow R_{0}\right.$, $R_{, t} \rightarrow 0$, and $R_{, t t} \rightarrow 0$ ), we find that $\eta_{\infty}>0$ and $\xi_{\infty}<0$, so that a spherical bubble will be stable under all perturbation modes. Under these conditions, we can also obtain the natural frequency of vibration $\omega_{l}$ for a bubble in a viscoelastic material corresponding to each non-spherical

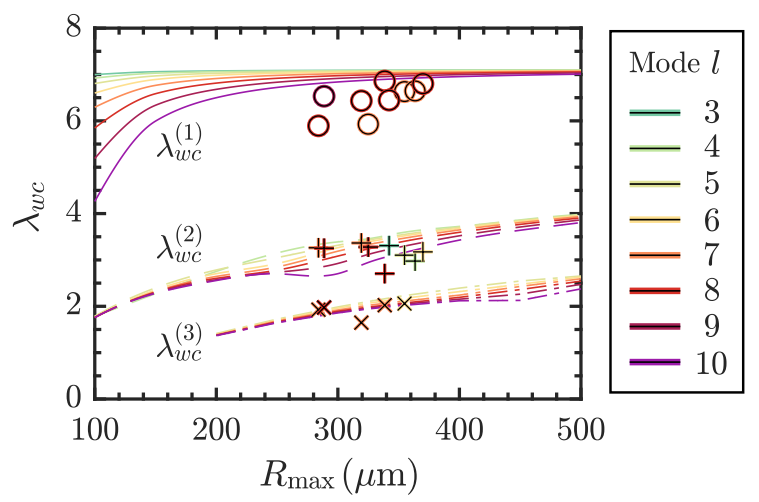

FIG. 4. Theoretically predicted and experimentally measured values of the critical hoop stress at the bubble wall $\lambda_{w c}$ at the onset of instability during the first three collapse cycles as a function of the maximum bubble radius $R_{\max }$ and the nonspherical mode shape number $l$, where $\lambda_{\max }$ is fixed at the same value as in Fig. 2.

mode shape $l$ :

$$
\omega_{l}^{2}=\frac{4 G}{\rho R_{0}^{2}}+\frac{G l(l+1)}{3 \rho R_{0}^{2}}+\frac{(l+2)(l-1) \gamma}{\rho R_{0}^{3}} .
$$

As discussed in [18], a non-spherical mode becomes unstable under continuous external driving when the driving frequency $\omega_{d}$ equals $2 \omega_{l}$.

Taking a closer look at the temporal evolution of the experimentally measured bubble wall hoop stretch, $\lambda_{w}$ (Fig. 3(a)), we find that the onset of instabilities occurs for $\lambda_{w}>1$. This marks a significant departure from previous surface instability investigations under quasi-static loading conditions, where rugae patterns require a stretch ratio within the plane of the surface that is less than one [21, 27-30]. Intrigued by these observations, we ask how well our instability model predicts the emergence of instabilities for $\lambda_{w}>1$. In Fig. 4, we plot the theoretically predicted values of $\lambda_{w}$ at the onset of instability during the first three collapse cycles as a function of the maximum bubble radius $R_{\max }$ and the non-spherical mode shape number $l$ [31]. Next, using the experimentally measured values of $R_{\max }$, we plot the critical values of $\lambda_{w}$ at the onset of each experimentally observed instability occurring in Fig. 2, and we find that the critical values during the first three collapse cycles are $\lambda_{w c}^{(1)}=6.5 \pm 0.3$ (circles in Fig. 4), $\lambda_{w c}^{(2)}=3.2 \pm 0.2$ (pluses in Fig. 4), and $\lambda_{w c}^{(3)}=1.8 \pm 0.2$ (crosses in Fig. 4 ), respectively. Comparing the experimentally obtained values of $\lambda_{w c}$ with the ones predicted by our instability model, we see good agreement across all mode shapes, as shown in Fig. 4.

It is important to note that other types of nonspherical instabilities are also possible during an inertial cavitation event. For example, in Fig. 1(c-vii), one can see crease-like instabilities near the bubble wall for 
$\lambda_{w}<1$ [32-34]. Axisymmetric instabilities may also appear during inertial cavitation in heterogeneous media or in the vicinity of impedance mismatched boundaries [35-37]. The critical conditions for predicting the onset of these and other non-spherical instabilities remain an active area of research and will be the subject of future work.

Finally, through fully 3D finite element simulations, we find that non-spherical instabilities near the bubble wall can induce strain and stress amplification in soft materials, which might lead to a thin damage layer developing near the bubble wall [38]. It is also conceivable that within such a layer the material could experience inelastic deformation, fracture, or significant strain softening [39-41]. While addressing appropriate material damage models is an exciting research area beyond the scope presented here, we nevertheless hope that the results of our theory motivate future studies aimed at resolving the intricate mechanics and physics near the bubble wall during these high strain-rate, inertially dominated deformations.

In sum, this paper presents a new theoretical framework for predicting the dynamic onset and evolution of complex non-spherical instability shapes in nonlinear viscoelastic soft materials during inertial cavitation, and provides a new foundation for characterizing and classifying dynamic instabilities under extreme loading conditions.

We gratefully acknowledge funding support from the Office of Naval Research (Dr. Timothy Bentley) under grant N000141712058. We thank Dr. Lauren Hazlett for helpful discussions and editing of the manuscript.

* cfranck@wisc.edu; Corresponding author

[1] M. S. Plesset, J. App. Mech. 25, 96 (1954).

[2] A. Prosperetti, Q. App. Math. 34, 339 (1977).

[3] J. C. Luo, H. Ching, B. G. Wilson, A. Mohraz, E. L. Botvinick, and V. Venugopalan, Sci. Rep. 10, 13144 (2020).

[4] C. W. Barney, C. E. Dougan, K. R. McLeod, A. KazemiMoridani, Y. Zheng, Z. Ye, S. Tiwari, I. Sacligil, R. A. Riggleman, S. Cai, J.-H. Lee, S. R. Peyton, G. N. Tew, and A. J. Crosby, Proc. Natl. Acad. Sci. U.S.A. 117, 9157 (2020).

[5] B. Saint-Michel and V. Garbin, Curr. Opin. Colloid. In. , 101392 (2020).

[6] J. B. Keller and M. Miksis, J. Acoust. Soc. Am. 68, 628 (1980).

[7] J. B. Estrada, C. Barajas, D. L. Henann, E. Johnsen, and C. Franck, J. Mech. Phy. Solids 112, 291 (2018).

[8] J. Yang, H. C. Cramer, and C. Franck, Extrem. Mech. Lett. 39, 100839 (2020).

[9] M. P. Brenner, D. Lohse, and T. F. Dupont, Phys. Rev. Lett. 75, 954 (1995).

[10] F. Hamaguchi and K. Ando, Phys. Fluids. 27, 113103 (2015).
[11] S. J. Shaw, Phys. Fluids. 29, 122103 (2017).

[12] M. Guédra and C. Inserra, J. Fluid. Mech. 857, 681 (2018).

[13] B. Saint-Michel and V. Garbin, Soft Matter (2020).

[14] H. Y. Yoshikawa, R. Murai, H. Adachi, S. Sugiyama, M. Maruyama, Y. Takahashi, K. Takano, H. Matsumura, T. Inoue, S. Murakami, H. Masuhara, and Y. Mori, Chem. Soc. Rev. 43, 2147 (2014).

[15] R. Mettin, C. Cairós, and A. Troia, Ultrason. Sonochem. 25, 24 (2015).

[16] C. E. Brennen, Interface focus 5, 20150022 (2015).

[17] G. I. Taylor, Proc. R. Soc. London A 201, 192 (1950).

[18] K. Murakami, R. Gaudron, and E. Johnsen, Ultrasonics Sonochemistry 67, 105170 (2020).

[19] R. Gaudron, K. Murakami, and E. Johnsen, J. Mech. Phys. Solids. 143, 104047 (2020).

[20] Upper and lower bounds, mean, and RMS values of the magnitude of bubble surface instabilities are summarized in Supplementary Material S2.

[21] M. Diab, T. Zhang, R. Zhao, H. Gao, and K.-S. Kim, Proc. R. Soc. A-Math. Phy. 469, 20120753 (2013).

[22] M. S. Plesset, J. App. Mech. 16, 277 (1949).

[23] L. Mancia, J. Yang, J.-S. Spratt, J. R. Sukovich, Z. Xu, T. Colonius, C. Franck, and E. Johnsen, Soft Matter (2021).

[24] C. Barajas and E. Johnsen, J. Acoust. Soc. Am. 141, 908 (2017).

[25] Derivation of the ansatz for the perturbed deformation is summarized in Supplementary Material S3.

[26] See Supplementary Material S5 for more information regarding the stability phase diagram for (7).

[27] S. Cai, K. Bertoldi, H. Wang, and Z. Suo, Soft Matter 6, 5770 (2010).

[28] L. Jin, S. Cai, and Z. Suo, Europhys. Lett. 95, 64002 (2011).

[29] B. Li, Y.-P. Cao, X.-Q. Feng, and H. Gao, Soft Matter 8, 5728 (2012).

[30] R. Zhao and X. Zhao, J. Appl. Mech. 84 (2017).

[31] The initial hoop stretch ratio $\lambda_{\max }$ is fixed at the same value as in Fig. 2. Details of the theoretical predictions are summarized in Supplementary Material S7.

[32] M. P. Milner, L. Jin, and S. B. Hutchens, Soft Matter 13, 6894 (2017).

[33] M. A. Bruning, M. Costalonga, J. H. Snoeijer, and A. Marin, Phys. Rev. Lett. 123, 214501 (2019).

[34] J. Yang, H. C. Cramer III, and C. Franck, in Dynamic Behavior of Materials in Conf. Proc. Soc. Exp. Mech. (2021).

[35] E.-A. Brujan, K. Nahen, P. Schmidt, and A. Vogel, J. Fluid. Mech. 433, 251 (2001).

[36] N. Bremond, M. Arora, S. M. Dammer, and D. Lohse, Phys. Fluids. 18, 121505 (2006).

[37] J. Yang, Y. Yin, H. Cramer III, and C. Franck, in Dynamic Behavior of Materials in Conf. Proc. Soc. Exp. Mech. (2022).

[38] Fully 3D finite element simulations are presented in Supplementary Material S8.

[39] S. B. Hutchens, S. Fakhouri, and A. J. Crosby, Soft Matter 12, 2557 (2016).

[40] P. Movahed, W. Kreider, A. D. Maxwell, S. B. Hutchens, and J. B. Freund, J. Acoust. Soc. Am. 140, 1374 (2016).

[41] S. Raayai-Ardakani, D. R. Earl, and T. Cohen, Soft Matter 15, 4999 (2019). 


\title{
SUPPLEMENTARY MATERIAL of "Predicting complex Instability Shapes of Inertial Cavitation Bubbles in Viscoelastic Soft Matter"
}

\author{
Jin Yang, ${ }^{1}$ Anastasia Tzoumaka, ${ }^{2}$ Kazuya Murakami, ${ }^{3}$ Eric Johnsen, ${ }^{3}$ David L. Henann, ${ }^{2}$ and Christian Franck ${ }^{1, *}$ \\ ${ }^{1}$ Department of Mechanical Engineering, University of Wisconsin-Madison \\ ${ }^{2}$ School of Engineering, Brown University \\ ${ }^{3}$ Department of Mechanical Engineering, University of Michigan, Ann Arbor
}

(Dated: April 8, 2021)

\section{S1. POLYACRYLAMIDE HYDROGEL PREPARATION}

To prepare polyacrylamide (PA) hydrogels, $40.0 \%$ acrylamide solution and $2.0 \%$ bis solution (Bio-Rad, Hercules, $\mathrm{CA}$ ) are mixed to a final concentration of $8.0 \% / 0.08 \%$ Acrylamide/Bis (v/v) in deionized water and crosslinked with $0.5 \%$ Ammonium Persulfate (APS) and $1.25 \%$ N,N,N',N'-tetramethylethane-1,2-diamine (TEMED)(ThermoFisher Scientific, USA). Once mixed, PA samples were allowed to polymerize in custom $15 \mathrm{~mm}$ diameter by $2 \mathrm{~mm}$ height cylindrical delrin molds for 1 hour. PA hydrogels were then submerged in deionized water 24 hours before testing to allow for any swelling to equilibrate. The viscoelastic material properties of the final prepared PA hydrogels are characterized by a quadratic law strain stiffening Kelvin-Voigt (qKV) viscoelastic model with ground-state shear modulus $G=2.77 \mathrm{kPa}$, viscosity $\mu=0.186 \pm 0.194 \mathrm{~Pa} \cdot \mathrm{s}$, and strain stiffening parameter $\alpha=0.48 \pm 0.14$ [1].

\section{S2. MAGNITUDE OF BUBBLE SURFACE PERTURBATIONS}

This section describes how we quantitatively measure the magnitude of bubble surface perturbations in our experiments. First, we perform image post-processing to extract the outline of bubble wall from each video frame, see Fig. S1(a-b), which is then rewritten into a polar coordinate system $\{r, \phi\}$, see Fig. S1(c), where $r$ and $\phi$ are the radial and polar angular coordinates and defined in Fig. S1(b). We further define the mean radius of the bubble as:

$$
\bar{r}=\frac{1}{2 \pi} \int_{\phi} r d \phi
$$

The upper bound, lower bound, mean, and root-mean-square (RMS) values of the magnitude of the bubble surface perturbation are further defined as:

$$
\begin{aligned}
a_{\text {upper bound }} & =\max _{\phi}|r-\bar{r}|, \\
a_{\text {lower bound }} & =\min _{\phi}|r-\bar{r}|, \\
a_{\text {mean }} & =\frac{1}{2 \pi} \int_{\phi}|r-\bar{r}| d \phi, \\
a_{\text {RMS }} & =\left(\frac{1}{2 \pi} \int_{\phi}|r-\bar{r}|^{2} d \phi\right)^{1 / 2} .
\end{aligned}
$$

\section{S3. DERIVATION OF THE ANSATZ FOR THE PERTURBED DISPLACEMENT FIELD IN MAIN TEXT EQ (4)}

Consider a spherical void (see Fig. S2) with reference configuration $\mathcal{B}_{0}\left(r_{0}, \theta_{0}, \phi_{0}\right),\left\{R_{0} \leqslant r_{0} \leqslant \infty, 0 \leqslant \theta_{0} \leqslant \pi, 0 \leqslant\right.$ $\left.\phi_{0} \leqslant 2 \pi\right\}$, and current deformed configuration $\mathcal{B}(r, \theta, \phi),\{R \leqslant r \leqslant \infty, 0 \leqslant \theta \leqslant \pi, 0 \leqslant \phi \leqslant 2 \pi\}$, where $\left\{r_{0}, r\right\}$

\footnotetext{
* cfranck@wisc.edu; Corresponding author
} 
(a)

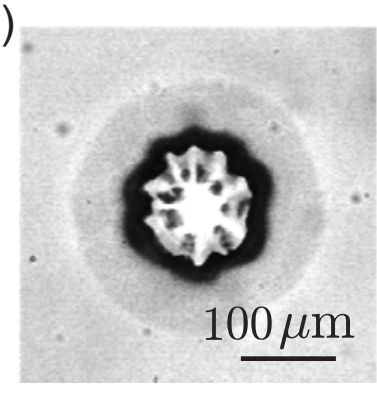

(b)

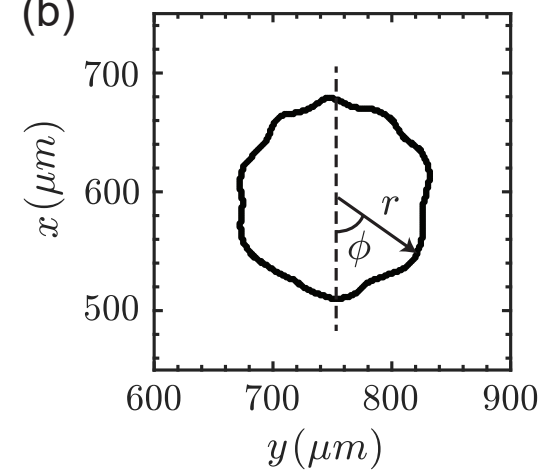

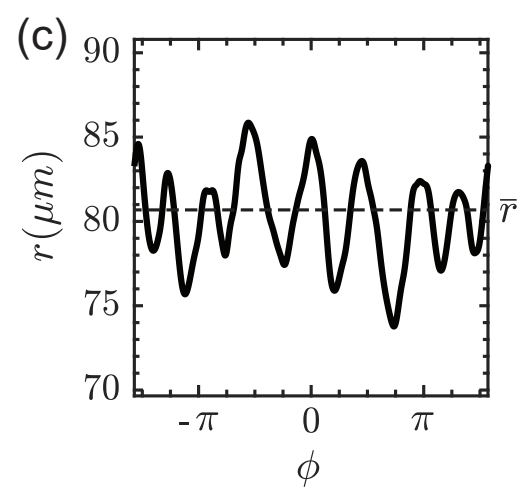

Fig. S1. Measurement of magnitude of bubble shape instabilities. (a) A typical frame from the recorded experimental video. (b) Bubble shape is extracted from the image post-processing of each frame. (c) Bubble surface is further transformed into a polar coordinate system.

represent referential and current radial coordinates, $\left\{\phi_{0}, \phi\right\}$ are referential and current azimuthal angular coordinates, and $\left\{\theta_{0}, \theta\right\}$ are referential and current polar angular coordinates. The time-dependent bubble radius is $R(t)$, and $R_{0}$ denotes the undeformed bubble radius. The spherically symmetric base state of deformation is described by the radial mapping $r=\left(r_{0}^{3}+R^{3}(t)-R_{0}^{3}\right)^{1 / 3}$, so that in the base state, the only non-zero component of displacement is $u_{r}=r-r_{0}$. We then consider a displacement perturbation of the form $\left\{\tilde{u}_{r}, \tilde{u}_{\theta}, \tilde{u}_{\phi}\right\}$, which is added to the base-state displacement field. The resulting deformation gradient is $\mathbf{F}=\mathbf{F}_{0}+\mathbf{d}$, where $\mathbf{F}_{0}$ is the base-state deformation gradient, which can be expressed referentially as

$$
\left[\mathbf{F}_{0}\right]=\left[\begin{array}{ccc}
\frac{r_{0}^{2}}{\left(r_{0}^{3}+R^{3}-R_{0}^{3}\right)^{2 / 3}} & 0 & 0 \\
0 & \frac{\left(r_{0}^{3}+R^{3}-R_{0}^{3}\right)^{1 / 3}}{r_{0}} & 0 \\
0 & 0 & \frac{\left(r_{0}^{3}+R^{3}-R_{0}^{3}\right)^{1 / 3}}{r_{0}}
\end{array}\right] \quad \text { so that } \quad \operatorname{det} \mathbf{F}_{0}=1
$$

and $\mathbf{d}$ is the perturbation of the deformation gradient, which can be expressed spatially as

$$
[\mathbf{d}]=\left[\begin{array}{ccc}
\frac{\partial \tilde{u}_{r}}{\partial r} & \frac{1}{r} \frac{\partial \tilde{u}_{r}}{\partial \theta}-\frac{\tilde{u}_{\theta}}{r} & \frac{1}{r \sin \theta} \frac{\partial \tilde{u}_{r}}{\partial \phi}-\frac{\tilde{u}_{\phi}}{r} \\
\frac{\partial \tilde{u}_{\theta} \theta}{\partial r} & \frac{1}{r} \frac{\partial \tilde{u}_{\theta}}{\partial \theta}+\frac{\tilde{u}_{r}}{r} & \frac{1}{r \sin \theta} \frac{\partial \tilde{u}_{\theta}}{\partial \phi}-\frac{\tilde{u}_{\phi} \cot \theta}{r} \\
\frac{\partial \tilde{u}_{\phi}}{\partial r} & \frac{1}{r} \frac{\partial \tilde{u}_{\phi}}{\partial \theta} & \frac{1}{r \sin \theta} \frac{\partial \tilde{u}_{\phi}}{\partial \phi}+\frac{\tilde{u}_{r}}{r}+\frac{\tilde{u}_{\theta} \cot \theta}{r}
\end{array}\right]
$$

Since the surrounding material is assumed to be incompressible, the incremental deformation gradient $\mathbf{d}$ must satisfy

$$
\operatorname{tr}(\mathbf{d})=\frac{\partial \tilde{u}_{r}}{\partial r}+\frac{1}{r} \frac{\partial \tilde{u}_{\theta}}{\partial \theta}+\frac{\tilde{u}_{r}}{r}+\frac{1}{r \sin \theta} \frac{\partial \tilde{u}_{\phi}}{\partial \phi}+\frac{\tilde{u}_{r}}{r}+\frac{\tilde{u}_{\theta} \cot \theta}{r}=0
$$

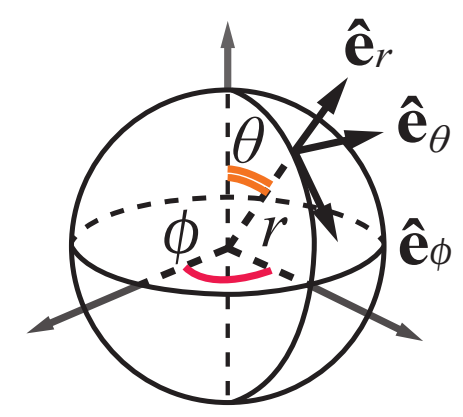

Fig. S2. Spherical coordinates $\{r, \theta, \phi\}$. 
We consider the following displacement perturbation:

$$
\left\{\begin{array}{l}
\tilde{u}_{r}=f(r) \cos (m \phi) P_{l}^{m}(\cos \theta) \\
\tilde{u}_{\theta}=g(r) \cos (m \phi) \frac{d}{d \theta} P_{l}^{m}(\cos \theta) \\
\tilde{u}_{\phi}=h(r) m \sin (m \phi) P_{l}^{m}(\cos \theta)
\end{array}\right.
$$

where $f(r), g(r)$, and $h(r)$ are functions that only depend on the spatial radial coordinate $r$. Perturbations of this form are related to the normalized spherical harmonic functions $Y_{l}^{m}(\theta, \phi):=\cos (m \phi) P_{l}^{m}(\cos \theta)$, where $P_{l}^{m}(\cos \theta)$ are the normalized associated Legendre polynomials, $m$ is the fixed wavenumber in the azimuthal angular coordinate, and the integers $m$ and $l$ satisfy $|m| \leqslant l$.

After inserting the ansatz (S9) into the incompressibility condition (S8), we obtain

$$
\frac{\partial f}{\partial r}+\frac{2 f}{r}-\frac{g}{r}\left[l(l+1)-\frac{m^{2}}{\sin ^{2} \theta}\right]+\frac{m^{2} h}{r \sin \theta}=0 .
$$

Here, motivated by our experimental observations, we focus on non-spherical, non-axisymmetric instabilities, in which $m>0$. Thus, the solution of Eq. (S10) obeys following conditions:

$$
\begin{aligned}
& g=h=0 \quad \Rightarrow \quad \tilde{u}_{\theta}=\tilde{u}_{\phi}=0, \\
& \frac{\partial f}{\partial r}+\frac{2 f}{r}=0 \Rightarrow f(r)=C r^{-2} .
\end{aligned}
$$

We further rewrite $C$ as $a R^{2}$, where $a(t)$ is the time-dependent perturbation magnitude at the bubble wall, so that (S9) takes the form of Eq. (4) in the main text.

\section{S4. DERIVATION OF EQS (7-8) IN THE MAIN TEXT}

\section{A. Kinematics}

First, we calculate several kinematical fields in the surrounding soft material. For the ansatz for the perturbed displacement field in main text Eq. (4), the deformation mapping is

$$
\left\{\begin{array}{l}
r\left(r_{0}, \theta_{0}, \phi_{0}, t\right)=\left(r_{0}^{3}+R^{3}(t)-R_{0}^{3}\right)^{1 / 3}+\frac{a(t) R^{2}(t)}{\left(r_{0}^{3}+R^{3}(t)-R_{0}^{3}\right)^{2 / 3}} Y_{l}^{m}\left(\theta_{0}, \phi_{0}\right), \\
\theta\left(r_{0}, \theta_{0}, \phi_{0}, t\right)=\theta_{0} \\
\phi\left(r_{0}, \theta_{0}, \phi_{0}, t\right)=\phi_{0}
\end{array}\right.
$$

where $Y_{l}^{m}(\theta, \phi)$ is the normalized $(l, m)^{t h}$ spherical harmonic function, and $a(t)$ is a time-dependent coefficient, which is assumed to be much smaller than $R(t)$. The referential Lagrangian description of the displacement field is then

$$
\left\{\begin{array}{l}
u_{r}\left(r_{0}, \theta_{0}, \phi_{0}, t\right)=\left(r_{0}^{3}+R^{3}(t)-R_{0}^{3}\right)^{1 / 3}-r_{0}+\frac{a(t) R^{2}(t)}{\left(r_{0}^{3}+R^{3}(t)-R_{0}^{3}\right)^{2 / 3}} Y_{l}^{m}\left(\theta_{0}, \phi_{0}\right), \\
u_{\theta}\left(r_{0}, \theta_{0}, \phi_{0}, t\right)=0 \\
u_{\phi}\left(r_{0}, \theta_{0}, \phi_{0}, t\right)=0
\end{array}\right.
$$

The deformation gradient tensor in the referential Lagrangian description can be simplified as

$$
\begin{aligned}
{[\mathbf{F}] } & =\left[\begin{array}{ccc}
\frac{\partial r}{\partial r_{0}} \frac{1}{r_{0}} \frac{\partial u_{r}}{\partial \theta_{0}} & \frac{1}{r_{0} \sin \theta_{0}} \frac{\partial u_{r}}{\partial \phi_{0}} \\
0 & \frac{r}{r_{0}} & 0 \\
0 & 0 & \frac{r}{r_{0}}
\end{array}\right] \\
& =\left[\begin{array}{cccc}
\frac{r_{0}^{2}}{\left(r_{0}^{3}+R^{3}-R_{0}^{3}\right)^{2 / 3}}-\frac{2 r_{0}^{2} a R^{2} Y_{l}^{m}}{\left(r_{0}^{3}+R^{3}-R_{0}^{3}\right)^{5 / 3}} & \frac{a R^{2}}{r_{0}\left(r_{0}^{3}+R^{3}-R_{0}^{3}\right)^{2 / 3}} \frac{\partial Y_{l}^{m}}{\partial \theta_{0}} & \frac{a R^{2}}{r_{0} \sin \theta_{0}\left(r_{0}^{3}+R^{3}-R_{0}^{3}\right)^{2 / 3}} \frac{\partial Y_{l}^{m}}{\partial \phi_{0}} \\
0 & \frac{\left(r_{0}^{3}+R^{3}-R_{0}^{3}\right)^{1 / 3}}{r_{0}}+\frac{a R^{2} Y_{l}^{m}}{r_{0}\left(r_{0}^{3}+R^{3}-R_{0}^{3}\right)^{2 / 3}} & 0 \\
0 & 0 & \frac{\left(r_{0}^{3}+R^{3}-R_{0}^{3}\right)^{1 / 3}}{r_{0}}+\frac{a R^{2} Y_{l}^{m}}{r_{0}\left(r_{0}^{3}+R^{3}-R_{0}^{3}\right)^{2 / 3}}
\end{array}\right] .
\end{aligned}
$$


Since $a(t) \ll R(t)$, the left Cauchy-Green deformation tensor can be linearized as

$$
\begin{aligned}
{[\mathbf{B}] } & =[\mathbf{F}][\mathbf{F}]^{\top} \\
& \approx\left[\begin{array}{ccc}
\frac{r_{0}^{4}}{\left(r_{0}^{3}+R^{3}-R_{0}^{3}\right)^{4 / 3}}-\frac{4 r_{0}^{4} a R^{2} Y_{l}^{m}}{\left(r_{0}^{3}+R^{3}-R_{0}^{3}\right)^{7 / 3}} & \frac{a R^{2}}{r_{0}^{2}\left(r_{0}^{3}+R^{3}-R_{0}^{3}\right)^{1 / 3}} \frac{\partial Y_{l}^{m}}{\partial \theta_{0}} & \frac{a R^{2}}{r_{0}^{2} \sin \theta_{0}\left(r_{0}^{3}+R^{3}-R_{0}^{3}\right)^{1 / 3}} \frac{\partial Y_{l}^{m}}{\partial \phi_{0}} \\
\frac{a R^{2}}{r_{0}^{2}\left(r_{0}^{3}+R^{3}-R_{0}^{3}\right)^{1 / 3}} \frac{\partial Y_{l}^{m}}{\partial \theta_{0}} & \frac{\left.a r_{0}^{3}+R^{3}-R_{0}^{3}\right)^{2 / 3}}{r_{0}^{2}}+\frac{2 a R^{2} Y_{l}^{m}}{r_{0}^{2}\left(r_{0}^{3}+R^{3}-R_{0}^{3}\right)^{1 / 3}} & 0 \\
\frac{a R^{2}}{r_{0}^{2} \sin \theta_{0}\left(r_{0}^{3}+R^{3}-R_{0}^{3}\right)^{1 / 3}} \frac{\partial Y_{l}^{m}}{\partial \phi_{0}} & 0 & \frac{\left(r_{0}^{3}+R^{3}-R_{0}^{3}\right)^{2 / 3}}{r_{0}^{2}}+\frac{2 a R^{2} Y_{l}^{m}}{r_{0}^{2}\left(r_{0}^{3}+R^{3}-R_{0}^{3}\right)^{1 / 3}}
\end{array}\right] .
\end{aligned}
$$

To express kinematical fields using a spatial description, we linearize the inverse deformation mapping:

$$
r_{0}(r, \theta, \phi, t) \approx\left(r^{3}+R_{0}^{3}-R^{3}(t)\right)^{1 / 3}-\frac{a(t) R^{2}(t)}{\left(r^{3}+R_{0}^{3}-R^{3}(t)\right)^{2 / 3}} Y_{l}^{m}(\theta, \phi) .
$$

Then, using the inverse mapping and linearizing, the left Cauchy-Green deformation tensor can be expressed spatially as

$$
[\mathbf{B}] \approx\left[\begin{array}{ccc}
\frac{\left(r^{3}+R_{0}^{3}-R^{3}\right)^{4 / 3}}{r^{4}}-\frac{4 a R^{2}\left(r^{3}+R_{0}^{3}-R^{3}\right)^{1 / 3} Y_{l}^{m}}{r^{4}} & \frac{a R^{2}}{r\left(r^{3}+R_{0}^{3}-R^{3}\right)^{2 / 3}} \frac{\partial Y_{l}^{m}}{\partial \theta} & \frac{a R^{2}}{r \sin \theta\left(r^{3}+R_{0}^{3}-R^{3}\right)^{2 / 3}} \frac{\partial Y_{l}^{m}}{\partial \phi} \\
\frac{a R^{2}}{r\left(r^{3}+R_{0}^{3}-R^{3}\right)^{2 / 3}} \frac{\partial Y_{l}^{m}}{\partial \theta} & \frac{r^{2}}{\left(r^{3}+R_{0}^{3}-R^{3}\right)^{2 / 3}}+\frac{2 a R^{2} r^{2} Y_{l}^{m}}{\left(r^{3}+R_{0}^{3}-R^{3}\right)^{5 / 3}} & 0 \\
\frac{a R^{2}}{r \sin \theta\left(r^{3}+R_{0}^{3}-R^{3}\right)^{2 / 3}} \frac{\partial Y_{l}^{m}}{\partial \phi} & 0 & \frac{r^{2}}{\left(r^{3}+R_{0}^{3}-R^{3}\right)^{2 / 3}}+\frac{2 a R^{2} r^{2} Y_{l}^{m}}{\left(r^{3}+R_{0}^{3}-R^{3}\right)^{5 / 3}}
\end{array}\right] .
$$

The referential description of the radial velocity is

$$
V_{r}\left(r_{0}, \theta_{0}, \phi_{0}, t\right)=\left.\frac{\partial u_{r}}{\partial t}\right|_{r_{0}}=\frac{R^{2} R_{, t}}{\left(r_{0}^{3}+R^{3}-R_{0}^{3}\right)^{2 / 3}}-\frac{2 a R^{4} R_{, t} Y_{l}^{m}}{\left(r_{0}^{3}+R^{3}-R_{0}^{3}\right)^{5 / 3}}+\frac{\left(a_{, t} R^{2}+2 a R R_{, t}\right) Y_{l}^{m}}{\left(r_{0}^{3}+R^{3}-R_{0}^{3}\right)^{2 / 3}}
$$

and upon linearization, the spatial description of the radial velocity is

$$
v_{r}(r, \theta, \phi, t) \approx \frac{R^{2} R_{, t}}{r^{2}}+\frac{a_{, t} R^{2}+2 a R R_{, t}}{r^{2}} Y_{l}^{m} .
$$

The spatial velocity gradient tensor is

$$
[\mathbf{L}]=[\nabla \mathbf{v}]=\left[\begin{array}{ccc}
-\frac{2 R^{2} R_{, t}}{r^{3}}-\frac{2\left(a, t R^{2}+2 a R R_{, t}\right)}{r^{3}} Y_{l}^{m} & \frac{\left(a, t R^{2}+2 a R R_{, t}\right)}{r^{3}} \frac{\partial Y_{l}^{m}}{\partial \theta} & \frac{\left(a_{, t} R^{2}+2 a R R, t\right)}{r^{3} \sin \theta} \frac{\partial Y_{l}^{m}}{\partial \phi} \\
0 & \frac{R^{2} R_{, t}}{r^{3}}+\frac{\left(a, t R^{2}+2 a R R, t\right.}{r^{3}} Y_{l}^{m} & 0 \\
0 & 0 & \frac{R^{2} R_{, t}}{r^{3}}+\frac{\left(a_{, t} R^{2}+2 a R R_{, t}\right)}{r^{3}} Y_{l}^{m}
\end{array}\right],
$$

and the rate of deformation tensor $\mathbf{D}$ is then

$$
\begin{aligned}
{[\mathbf{D}] } & =\frac{1}{2}\left([\mathbf{L}]+[\mathbf{L}]^{\top}\right) \\
& =\left[\begin{array}{ccc}
-\frac{2 R^{2} R_{, t}}{r^{3}}-\frac{2\left(a, t R^{2}+2 a R R_{, t}\right)}{r^{3}} Y_{l}^{m} & \frac{\left(a_{, t} R^{2}+2 a R R_{, t}\right)}{2 r^{3}} \frac{\partial Y_{l}^{m}}{\partial \theta} & \frac{\left(a_{, t} R^{2}+2 a R R_{, t}\right)}{2 r^{3} \sin \theta} \frac{\partial Y_{l}^{m}}{\partial \phi} \\
\frac{\left(a_{, t} R^{2}+2 a R R_{, t}\right)}{2 r^{3}} \frac{\partial Y_{l}^{m}}{\partial \theta} & \frac{R^{2} R, t}{r^{3}}+\frac{\left(a, t R^{2}+2 a R R_{, t}\right)}{r^{3}} Y_{l}^{m} & 0 \\
\frac{\left(a_{, t} R^{2}+2 a R R_{, t}\right)}{2 r^{3} \sin \theta} \frac{\partial Y_{l}^{m}}{\partial \phi} & 0 & \frac{R^{2} R_{, t}}{r^{3}}+\frac{\left(a_{, t} R^{2}+2 a R R_{, t}\right)}{r^{3}} Y_{l}^{m}
\end{array}\right] .
\end{aligned}
$$

The referential description of the radial acceleration is

$$
\begin{aligned}
A_{r}\left(r_{0}, \theta_{0}, \phi_{0}, t\right)=\left.\frac{\partial v_{r}}{\partial t}\right|_{r_{0}}= & \frac{2 R R_{, t}^{2}+R^{2} R_{, t t}}{\left(r_{0}^{3}+R^{3}-R_{0}^{3}\right)^{2 / 3}}-\frac{2 R^{4} R_{, t}^{2}}{\left(r_{0}^{3}+R^{3}-R_{0}^{3}\right)^{5 / 3}} \\
& +\frac{\left(a_{, t t} R^{2}+4 a_{, t} R R_{, t}+2 a R_{, t}^{2}+2 a R R_{, t t}\right)}{\left(r_{0}^{3}+R^{3}-R_{0}^{3}\right)^{2 / 3}} Y_{l}^{m} \\
& -\frac{2\left(2 a_{, t} R^{4} R_{, t}+6 a R^{3} R_{, t}^{2}+a R^{4} R_{, t t}\right)}{\left(r_{0}^{3}+R^{3}-R_{0}^{3}\right)^{5 / 3}} Y_{l}^{m}+\frac{10 a R^{6} R_{, t}^{2}}{\left(r_{0}^{3}+R^{3}-R_{0}^{3}\right)^{8 / 3}} Y_{l}^{m},
\end{aligned}
$$

and upon linearization, the spatial description of the radial acceleration is

$a_{r}(r, \theta, \phi, t)=\frac{2 R R_{, t}^{2}+R^{2} R_{, t t}}{r^{2}}-\frac{2 R^{4} R_{, t}^{2}}{r^{5}}+\frac{\left(a_{, t t} R^{2}+4 a_{, t} R R_{, t}+2 a R_{, t}^{2}+2 a R R_{, t t}\right)}{r^{2}} Y_{l}^{m}-\frac{\left(4 a_{, t} R^{4} R_{, t}+8 a R^{3} R_{, t}^{2}\right)}{r^{5}} Y_{l}^{m}$. 


\section{B. Momentum balance equation}

The momentum balance equation is

$$
\nabla \cdot \sigma=\rho \mathbf{a},
$$

where $\nabla \cdot(\bullet)$ is the spatial divergence operator; $\rho$ is the spatial mass density; $\mathbf{a}$ is the acceleration vector; and $\boldsymbol{\sigma}$ is the Cauchy stress tensor, which has the following explicit form for the quadratic law Kelvin-Voigt (qKV) material model:

$$
\boldsymbol{\sigma}=G\left[1+\alpha\left(I_{1}-3\right)\right] \mathbf{B}+2 \mu \mathbf{D}-p \mathbf{I},
$$

where $p$ is a pressure-like quantity arising due to the incompressibility constraint in the surrounding material, and $I_{1}$ is the first invariant of the left Cauchy-Green deformation tensor:

$$
I_{1}=\operatorname{tr}(\mathbf{B})=\frac{\left(r^{3}+R_{0}^{3}-R^{3}\right)^{4 / 3}}{r^{4}}+\frac{2 r^{2}}{\left(r^{3}+R_{0}^{3}-R^{3}\right)^{2 / 3}}-\frac{4 a R^{2}\left(r^{3}+R_{0}^{3}-R^{3}\right)^{1 / 3}}{r^{4}} Y_{l}^{m}+\frac{4 a R^{2} r^{2}}{\left(r^{3}+R_{0}^{3}-R^{3}\right)^{5 / 3}} Y_{l}^{m} .
$$

We note that the pressure-like quantity $p$ consists of the sum of the base-state field, which only depends on $r$, and a perturbation field, which depends on $r, \theta$, and $\phi$. In what follows, we focus on the radial component of the momentum balance equation (S25):

$$
\frac{\partial \sigma_{r r}}{\partial r}+\frac{1}{r} \frac{\partial \sigma_{r \theta}}{\partial \theta}+\frac{1}{r \sin \theta} \frac{\partial \sigma_{r \phi}}{\partial \phi}+\frac{2}{r}\left(\sigma_{r r}-\sigma_{\theta \theta}\right)+\frac{\cot \theta}{r} \sigma_{r \theta}=\rho a_{r},
$$

where we have recognized that $\sigma_{\theta \theta}=\sigma_{\phi \phi}$.

\section{Boundary conditions}

The traction boundary condition at the bubble wall $\left(r=R+a Y_{l}^{m}\right)$ is

$$
\left.\boldsymbol{\sigma} \mathbf{n}\right|_{r=R+a Y_{l}^{m}}=-p_{\mathrm{b}} \mathbf{n}-\gamma\left(\nabla_{\mathcal{S}} \cdot \mathbf{n}\right) \mathbf{n},
$$

where $[\mathbf{n}]=\left[-1, a Y_{l, \theta}^{m} / R, a Y_{l, \phi}^{m} /(R \sin \theta)\right]^{\top}$ is the linearized outward unit normal vector on the bubble wall, $p_{\mathrm{b}}$ is the pressure inside the bubble, and $\nabla_{\mathcal{S}} \cdot(\bullet)$ is the surface divergence operator in the deformed configuration. After linearization [2], the radial component of Eq. (S29) is

$$
\left.\sigma_{r r}\right|_{r=R+a Y_{l}^{m}}=-p_{b}+\frac{2 \gamma}{R}+\frac{(l+2)(l-1)}{R^{2}} a Y_{l}^{m} .
$$

In the far-field, the perturbation decays to zero, and the stress approaches a state of hydrostatic pressure: $\left.\boldsymbol{\sigma}\right|_{r \rightarrow \infty}=$ $-p_{\infty} \mathbf{I}$, where $p_{\infty}$ is the far-field atmospheric pressure. Therefore, the far-field boundary condition for the radial stress component is

$$
\left.\sigma_{r r}\right|_{r \rightarrow \infty}=-p_{\infty}
$$

\section{Results}

Using (S18) and (S22), we insert (S26) and (S24) into (S28), integrate over $r$ from the current, perturbed bubble wall $\left(R+a Y_{l}^{m}\right)$ to the far-field $(r \rightarrow \infty)$ and apply the radial boundary conditions (S30) and (S31). After collecting the $O(1)$ terms, we recover the Rayleigh-Plesset governing equation for the dynamics of the unperturbed bubble radius in a nonlinear strain-stiffening viscoelastic material, which is consistent with our previous study as shown in Yang, et al. [1]:

$$
R R_{, t t}+\frac{3}{2} R_{, t}^{2}=\frac{1}{\rho}\left(p_{b}-p_{\infty}+S-\frac{2 \gamma}{R}\right),
$$

where stress integral $S$ takes the form

$$
S=\frac{(3 \alpha-1) G}{2}\left[5-\left(\frac{R_{0}}{R}\right)^{4}-\frac{4 R_{0}}{R}\right]-\frac{4 \mu \dot{R}}{R}+2 \alpha G\left[\frac{27}{40}+\frac{1}{8}\left(\frac{R_{0}}{R}\right)^{8}+\frac{1}{5}\left(\frac{R_{0}}{R}\right)^{5}+\left(\frac{R_{0}}{R}\right)^{2}-\frac{2 R}{R_{0}}\right] .
$$


Next, collecting the $O(a)$ terms, we obtain the governing, second-order differential equation for the bubble-wall perturbation magnitude, $a$ :

$$
a_{, t t}+\eta a_{, t}-\xi a=0 ; \quad \eta=\frac{3 R_{, t}}{R}+\frac{4 \mu}{\rho R^{2}}+\frac{l(l+1) \mu}{3 \rho R^{2}},
$$

where $\xi$ explicitly accounts for inertial effects during cavitation, nonlinear deformations of the viscoelastic solid, and surface tension effects:

$$
\begin{aligned}
\xi= & -\frac{R_{, t t}}{R}+\frac{4 \mu R_{, t}}{\rho R^{3}}-\frac{2 l(l+1) \mu R_{, t}}{3 \rho R^{3}}-\frac{(l+2)(l-1) \gamma}{\rho R^{3}}-\frac{2 G R_{0}}{\rho R^{3}}\left(1+\frac{R_{0}^{3}}{R^{3}}\right)-\frac{G l(l+1)}{\rho\left(R^{2}+R R_{0}+R_{0}^{2}\right)} \\
& -\frac{2 \alpha G}{\rho R^{2}} \frac{\left(R-R_{0}\right)^{2}}{R R_{0}}\left(1+\frac{R_{0}}{R}\right)^{3}\left(2-\frac{2 R_{0}}{R}+\frac{3 R_{0}^{2}}{R^{2}}-\frac{R_{0}^{3}}{R^{3}}+\frac{R_{0}^{4}}{R^{4}}\right)-\frac{\alpha G l(l+1)\left(R-R_{0}\right)^{2}}{5 \rho R R_{0}\left(R^{2}+R R_{0}+R_{0}^{2}\right)}\left(10+\frac{6 R_{0}}{R}+\frac{3 R_{0}^{2}}{R^{2}}+\frac{R_{0}^{3}}{R^{3}}\right) .
\end{aligned}
$$

We note that the perturbation of the pressure-like quantity $p$ does not enter the derivation of Eqs. (S32)-(S35).

\section{S5. EIGENVALUES OF EQ (7) IN THE MAIN TEXT}

The second order ordinary differential equation for $a(t)$, Eq. (7) in the main text, can be rewritten as the following dynamical system:

$$
\frac{d}{d t}\left[\begin{array}{c}
a \\
a, t
\end{array}\right]=\left[\begin{array}{cc}
0 & 1 \\
\xi & -\eta
\end{array}\right]\left[\begin{array}{c}
a \\
a, t
\end{array}\right]
$$

It is straightforward to see that $\mathbf{a}^{*}:=\left\{a=0, a_{, t}=0\right\}$ is always an equilibrium point of (S36), and its stability depends on the sign of the eigenvalues of the dynamical system (S36). The eigenvalues are of the form

$$
\zeta_{1,2}=-\frac{\eta}{2} \pm \frac{\sqrt{\Delta}}{2} \quad \text { with } \quad \Delta=\eta^{2}+4 \xi
$$

which may be classified in the following cases:

- Case (i): If $\xi>0$, the eigenvalues are real and of opposite sign, and therefore, $\mathbf{a}^{*}$ is a saddle point (Fig. S3 $(\mathrm{a}-\mathrm{i}))$

- Case (ii): If $\xi<0, \eta<0$, and $\Delta>0$, the eigenvalues are real and positive, and $\mathbf{a}^{*}$ is a source-type instability point (Fig. S3 (a-ii));

- Case (iii): If $\xi<0, \eta<0$, and $\Delta<0$, the eigenvalues are complex with positive real part, and $\mathbf{a}^{*}$ is an unstable spiral-type instability (Fig. S3 (a-iii));

- Case (iv): If $\xi<0, \eta>0$, and $\Delta<0$, the eigenvalues are complex with negative real part, and $\mathbf{a}^{*}$ is a stable spiral (Fig. S3 (a-iv));

- Case (v): If $\xi<0, \eta>0$, and $\Delta>0$, the eigenvalues are real and negative, and $\mathbf{a}^{*}$ is a sink (Fig. S3 (a-v)).

A phase diagram summarizing the stability of the solution $\mathbf{a}^{*}=\left\{a=0, a_{, t}=0\right\}$ for an inertial cavitation bubble in a nonlinear viscoelastic medium (Eq (7) in the main text) is shown in Fig. S3(a).

\section{S6. ADDITIONAL RESULTS OF THE NUMERICAL SIMULATION IN FIG 3}

Here we provide additional details of the numerical simulations in Fig. 3 of the main text. We summarize the numerically simulated hoop stretch ratio at the bubble wall $\lambda_{w}$ and the variables $\Delta, \xi^{i}, \xi^{e}$, and $\xi^{s t}$ in Fig. S3(bf), where the variables $\xi^{i}, \xi^{e}, \xi^{s t}$ account for inertial and viscous effects during cavitation, nonlinear hyperelastic 
deformations of the surrounding solid, and surface tension effects, respectively:

$$
\begin{aligned}
\xi^{i}= & -\frac{R_{, t t}}{R}+\frac{4 \mu R_{, t}}{\rho R^{3}}+\frac{2 l(l+1) \mu R_{, t}}{3 \rho R^{3}}, \\
\xi^{e}= & -\frac{2 G R_{0}}{\rho R^{3}}\left(1+\frac{R_{0}^{3}}{R^{3}}\right)-\frac{G l(l+1)}{\rho\left(R^{2}+R R_{0}+R_{0}^{2}\right)} \\
& -\frac{2 \alpha G}{\rho R^{2}} \frac{\left(R-R_{0}\right)^{2}}{R R_{0}}\left(1+\frac{R_{0}}{R}\right)^{3}\left(2-\frac{2 R_{0}}{R}+\frac{3 R_{0}^{2}}{R^{2}}-\frac{R_{0}^{3}}{R^{3}}+\frac{R_{0}^{4}}{R^{4}}\right) \\
& -\frac{\alpha G l(l+1)\left(R-R_{0}\right)^{2}}{5 \rho R R_{0}\left(R^{2}+R R_{0}+R_{0}^{2}\right)}\left(10+\frac{6 R_{0}}{R}+\frac{3 R_{0}^{2}}{R^{2}}+\frac{R_{0}^{3}}{R^{3}}\right), \\
\xi^{s t}= & -\frac{(l+2)(l-1) \gamma}{\rho R^{3}} .
\end{aligned}
$$

Conditions in which non-spherical instabilities corresponding to a spherical harmonic function of mode shape 8 are predicted (i.e., $\eta<0$ or $\xi>0$ ) are marked as red shaded regions.

(a)

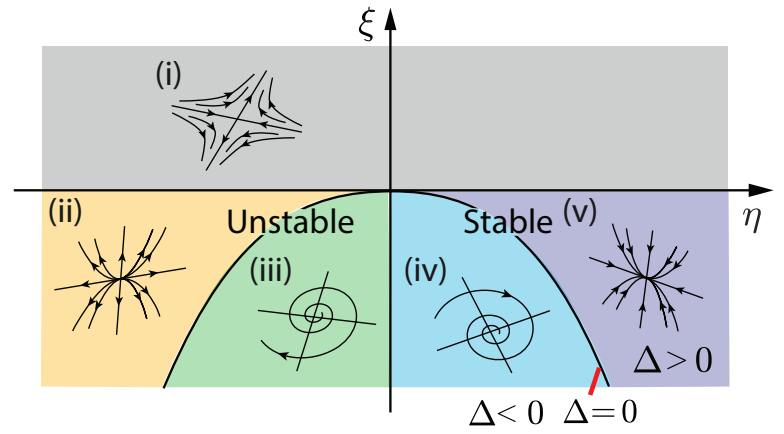

(b)
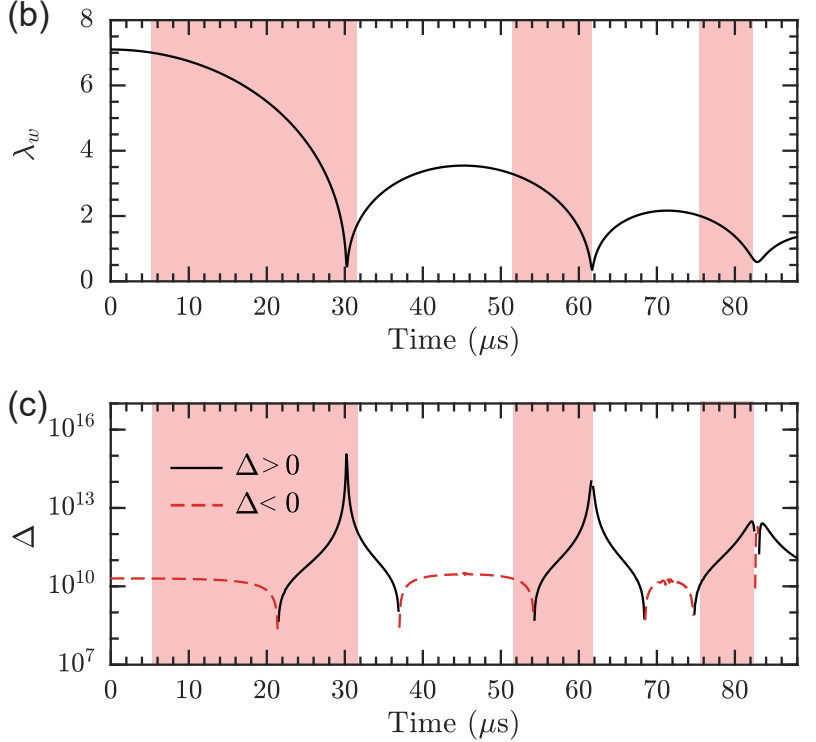

(d)

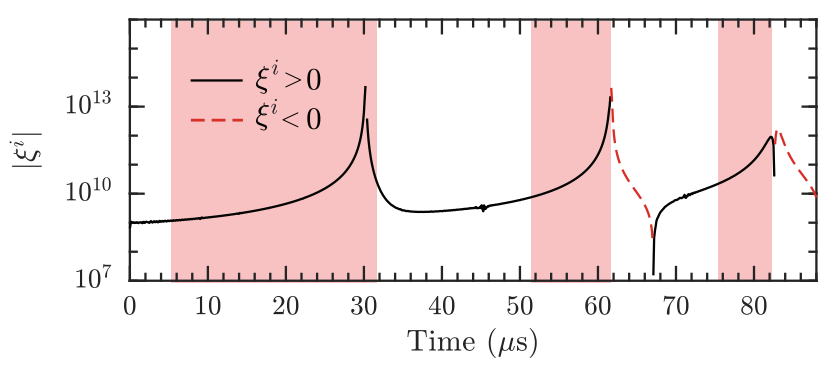

(e)
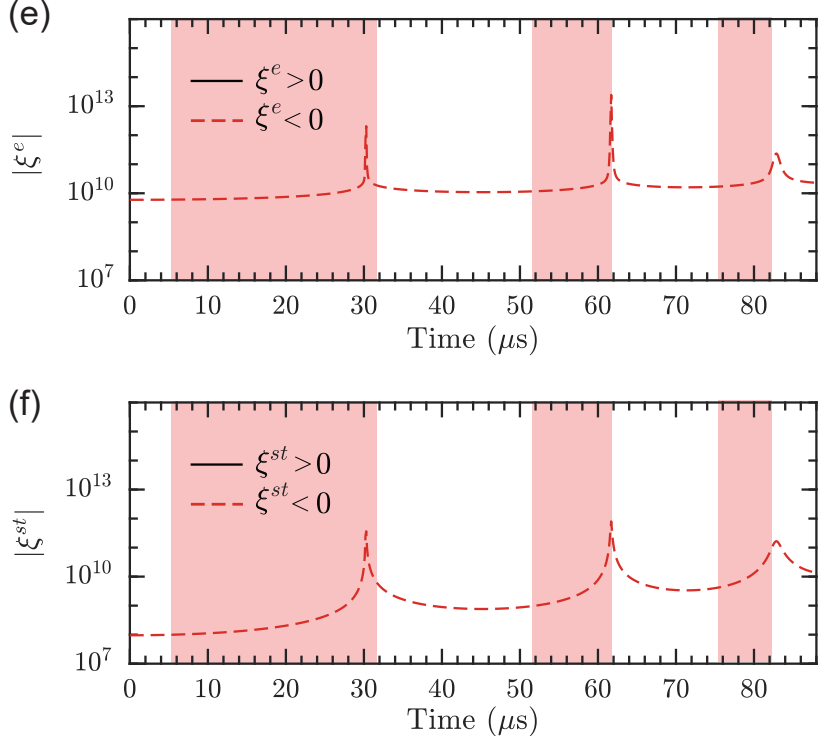

Fig. S3. (a) Phase diagram summarizing the stability of the solution $\mathbf{a}^{*}=\left\{a=0, a_{, t}=0\right\}$ for an inertial cavitation bubble in a nonlinear viscoelastic medium. Calculated (b) radial stretch ratio at the bubble wall $\lambda_{w}$, (c) $\Delta$, (d) $\xi^{i}$, (e) $\xi^{e}$, (f) $\xi^{s t}$ in the numerical simulation in Fig. 3 of the main text. Red shaded regions denote situations in which non-spherical instabilities corresponding to a spherical harmonic function of mode shape 8 are predicted.

\section{S7. DETAILS OF THEORETICAL PREDICTIONS IN FIG 4}

We theoretically predict the critical value of the radial stretch ratio at the bubble wall, $\lambda_{w}$, at the onset of bubble surface instability during the first three collapse cycles as a function of the maximum bubble radius $R_{\max }$ and the 
non-spherical mode shape number $l$. The initial $\lambda_{\max }$ is fixed at 7.1 , which is the same value as in all experimental tests shown in Figs. 1-2 in the main text. For each $R_{\max }$ value, we numerically solve the Rayleigh-Plesset equation, Eqs. (1) and (3), in the main text and compute the variables $\eta$ and $\xi$ using Eqs (7-8) in the main text. Then, we find the critical $\lambda_{w}$ at the onset of each instability mode occurring in the first three collapse cycles where $\eta<0$ or $\xi>0$, as shown in Figs. S4-S8, where the theoretically predicted non-spherical mode shape 8 instabilities are marked as red shaded regions. In Figs. S4-S8, $R_{\max }$ takes a value of $100 \mu m, 200 \mu m, 300 \mu m, 400 \mu m, 500 \mu m$, respectively.
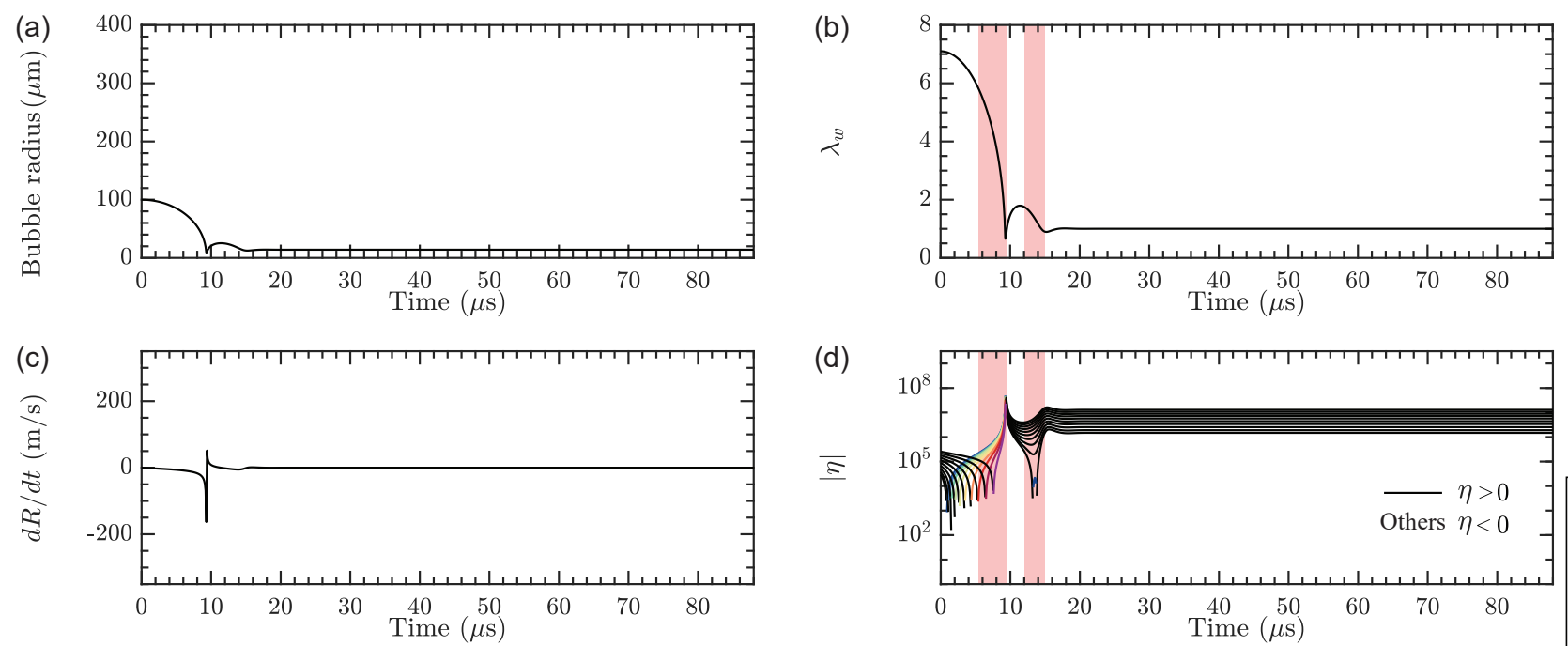

(d)
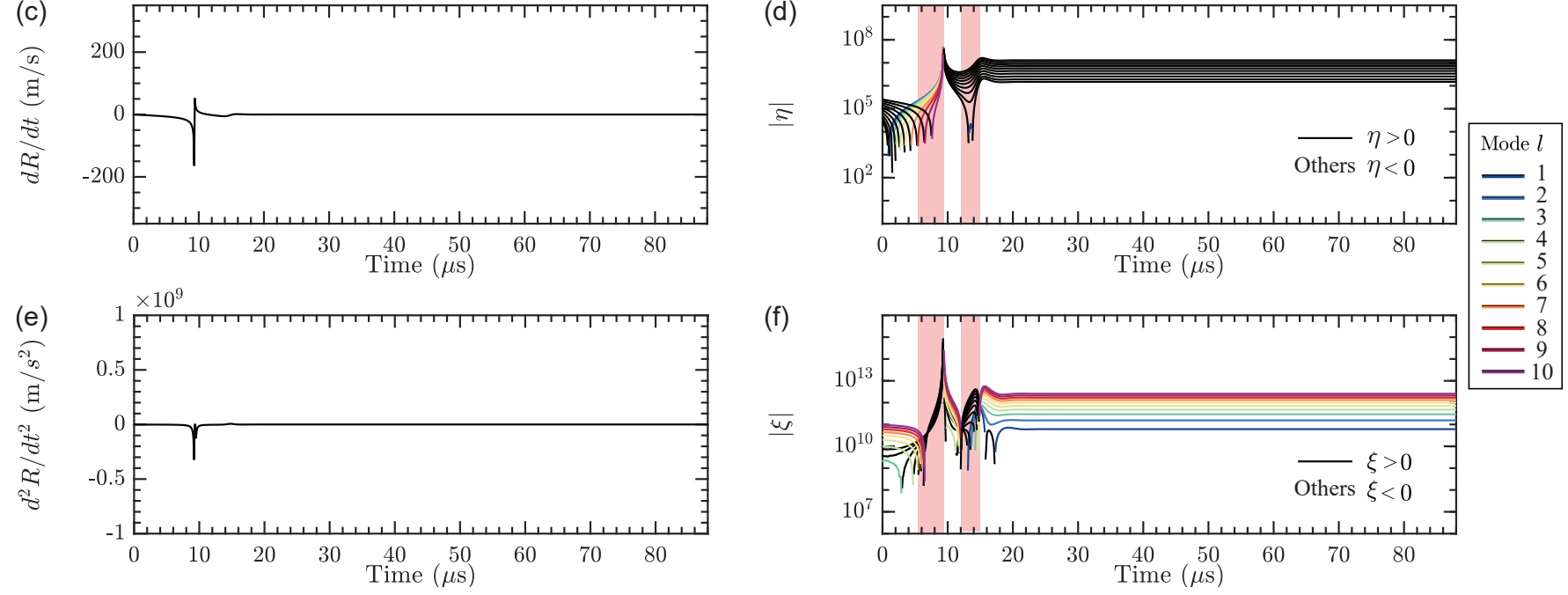

Fig. S4. (a,c,e) Numerically simulated bubble dynamics when $R_{\max }=100 \mu m$. (b,d,f) Calculated bubble wall stretch ratio $\lambda_{w}$ and the variables $\eta$ and $\xi$ calculated using Eqs (7-8) in the main text. Red shaded regions denote theoretically predicted non-spherical mode shape 8 instabilities.

\section{S8. FINITE ELEMENT NUMERICAL SIMULATIONS}

To examine the strain and stress fields induced by non-spherical deformation, we construct a finite-element model of an isolated 3D spherical cavity within an infinite material using the commercial software package Abaqus [3]. Using a user-material (UMAT) subroutine, we model the surrounding material as a quadratic law strain stiffening Kelvin-Voigt ( $\mathrm{qKV}$ ) viscoelastic material (Eq (2) in the main text) with shear modulus $G=2.77 \mathrm{kPa}$, strain stiffening parameter $\alpha=0.48$, and viscosity $\mu=0.186 \mathrm{~Pa} \cdot \mathrm{s}$. Due to the symmetry of the problem, we simulate one eighth of the sphere. The undeformed bubble radius is taken to be $45 \mu \mathrm{m}$, and the outer radius of the simulation domain is taken to be large enough so that an infinite surroundings is approximated. The mesh consists of 7275 3D, 8-node fully integrated hybrid elements. Next, to account for the non-spherical deformation, we impose a displacement boundary condition on the bubble wall surface consisting of the sum of a spherically symmetric deformation and a spherical harmonic perturbation (Eq. (4) in the main text), using a user-displacement (UDISP) subroutine. We perform a static simulation considering a time point between (iv) and (v) in the main text Fig. 1(a), corresponding to a deformed bubble radius of $72 \mu \mathrm{m}$, a perturbation amplitude of $1.6386 \mu \mathrm{m}$, and mode shape $l=8$.

In Fig. S9(a) and (b), we plot contours of the spherical component of the logarithmic strain, $E_{r r}$, while in Fig. S9(c) and (d) we plot contours of the circumferential component of the logarithmic strain $E_{\theta \theta}$. In Fig. S10(a) and (b) we plot contours of the spherical component of the Cauchy stress, $\sigma_{r r}$, while in Fig. S10(c) and (d) we plot contours of the circumferential component of the Cauchy stress, $\sigma_{\theta \theta}$. We include both the spherically symmetric case and the 

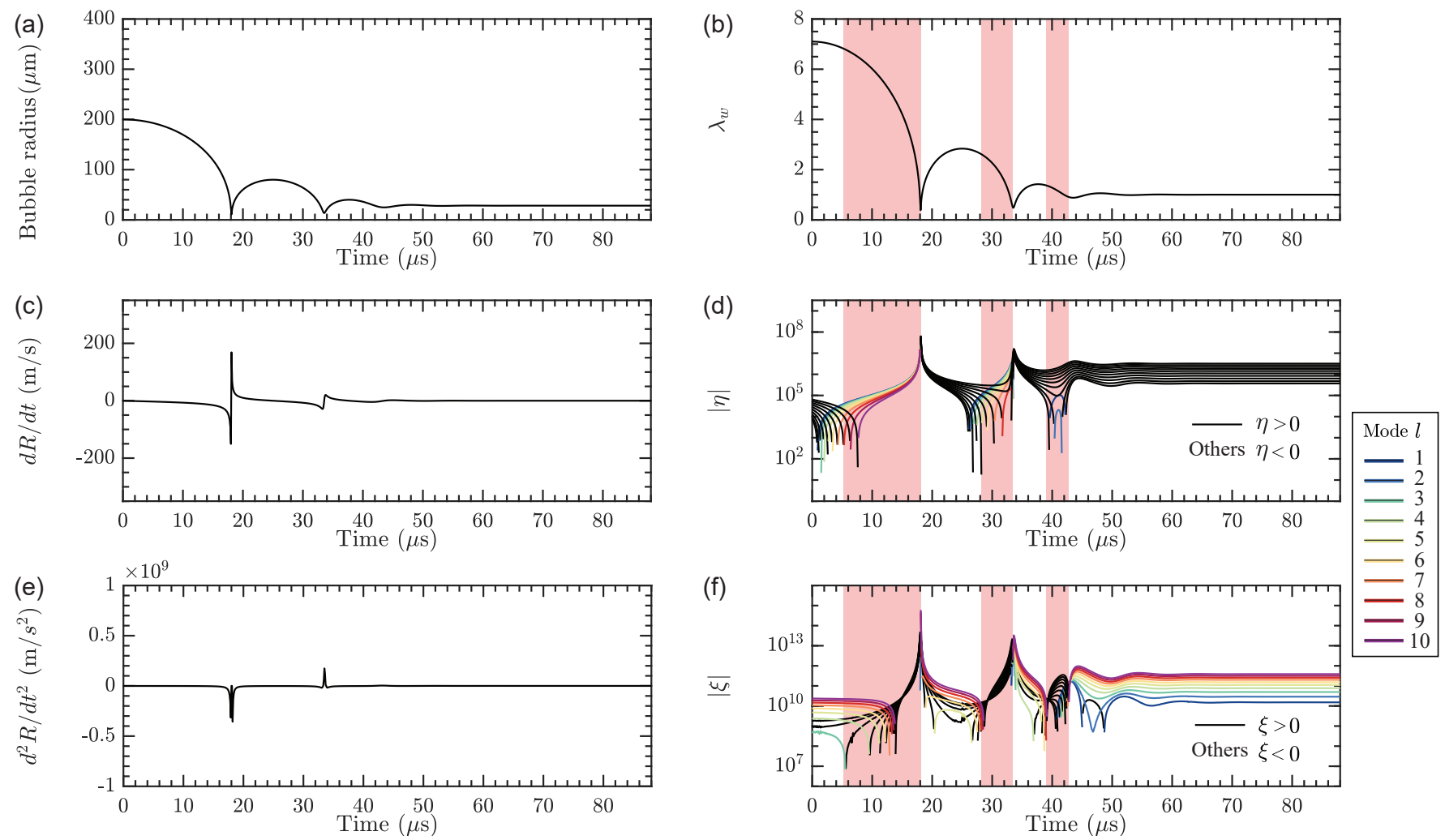

Fig. S5. (a,c,e) Numerically simulated bubble dynamics when $R_{\max }=200 \mu m$. (b,d,f) Calculated bubble wall stretch ratio $\lambda_{w}$ and the variables $\eta$ and $\xi$ calculated using Eqs (7-8) in the main text. Red shaded regions denote theoretically predicted non-spherical mode shape 8 instabilities.

perturbed case, illustrating the amplification of stress and strain due to non-spherical deformation, notably in the circumferential components of stress and strain.

[1] J. Yang, H. C. Cramer, and C. Franck, Extrem. Mech. Lett. 39, 100839 (2020).

[2] M. S. Plesset, J. App. Mech. 25, 96 (1954).

[3] Abaqus, "Reference manuals," Dassault Systèmes Simulia Corp. (2018). 


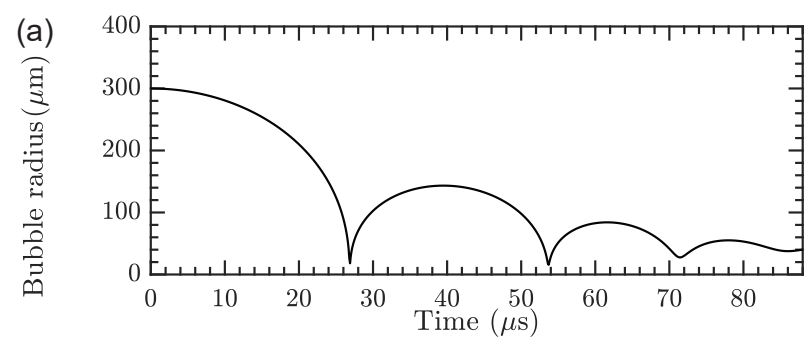

(b)

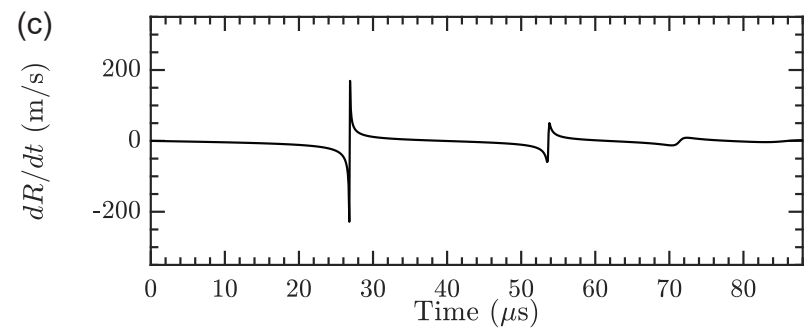

(d)
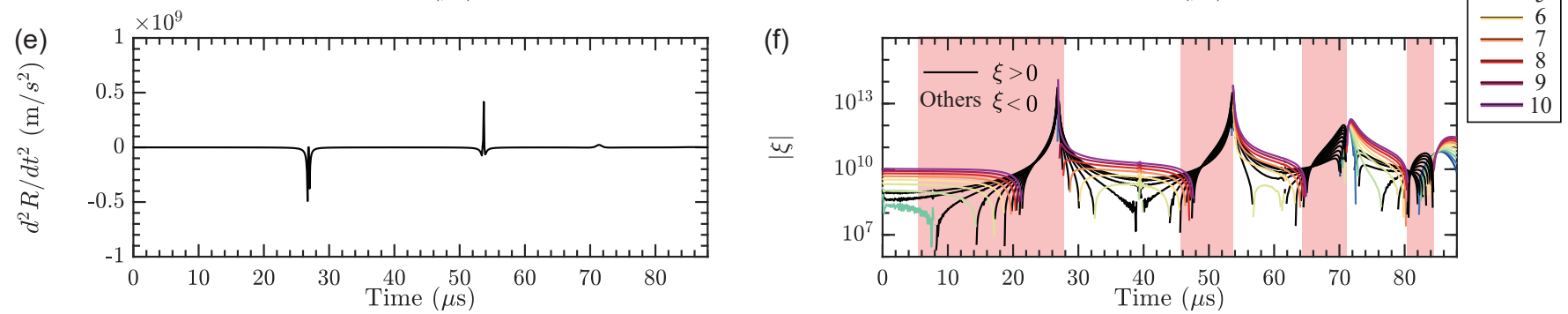

Fig. S6. (a,c,e) Numerically simulated bubble dynamics when $R_{\max }=300 \mu m$. (b,d,f) Calculated bubble wall stretch ratio $\lambda_{w}$ and the variables $\eta$ and $\xi$ calculated using Eqs (7-8) in the main text. Red shaded regions denote theoretically predicted non-spherical mode shape 8 instabilities. 

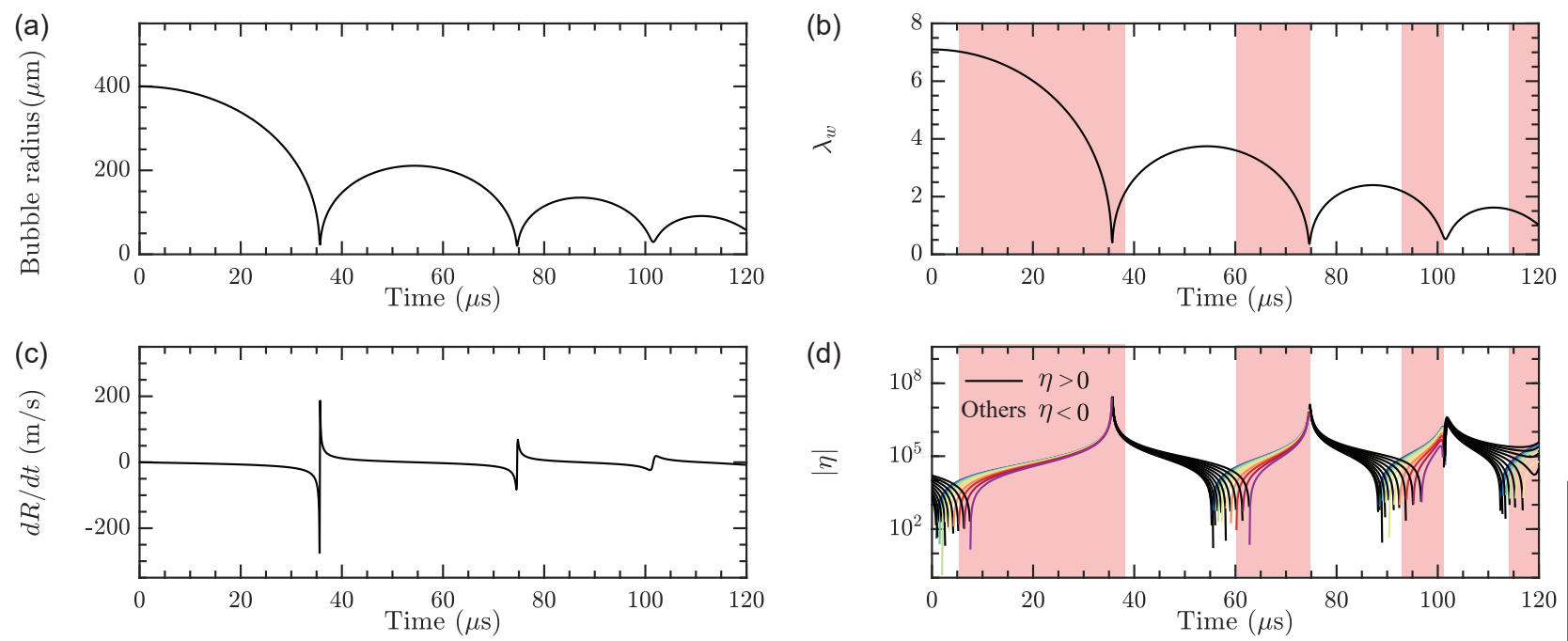

(d)
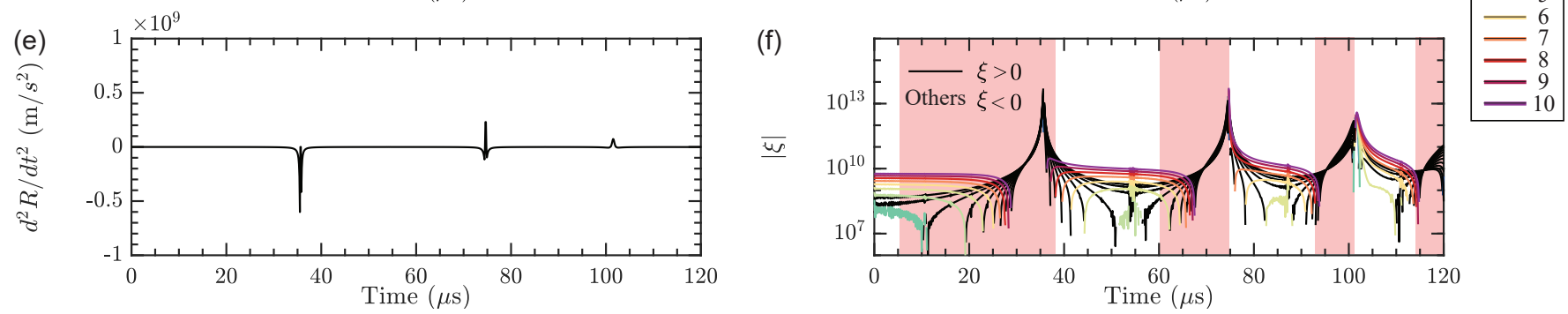

Fig. S7. (a,c,e) Numerically simulated bubble dynamics when $R_{\max }=400 \mu m$. (b,d,f) Calculated bubble wall stretch ratio $\lambda_{w}$ and the variables $\eta$ and $\xi$ calculated using Eqs (7-8) in the main text. Red shaded regions denote theoretically predicted non-spherical mode shape 8 instabilities. 

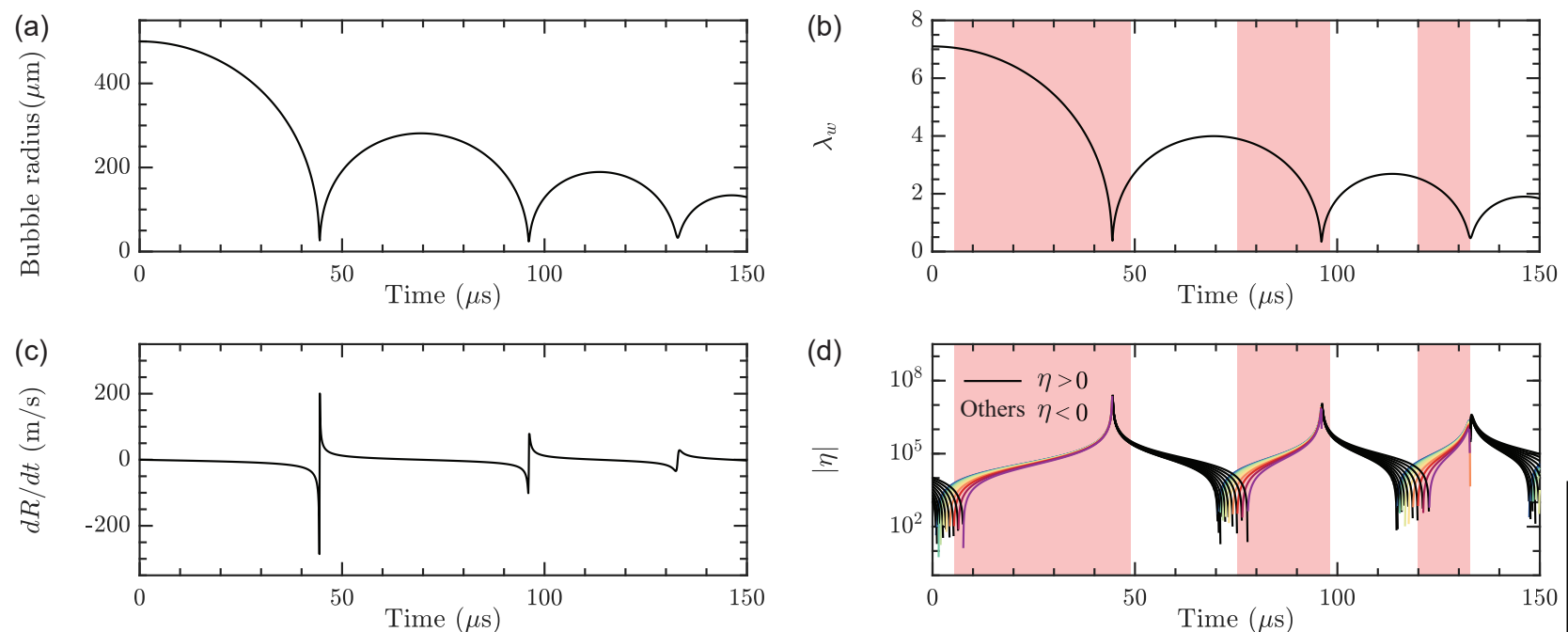

(d)

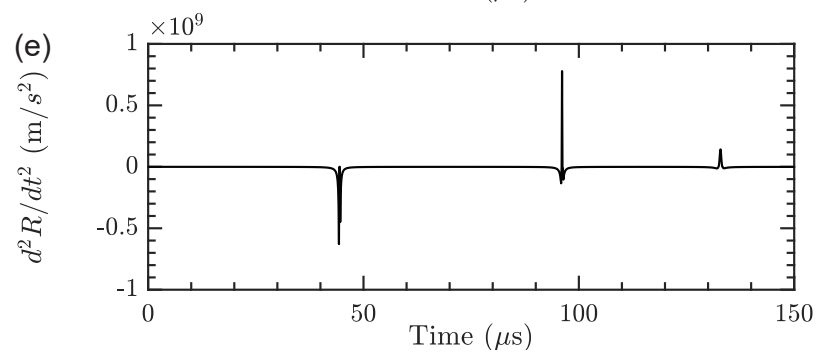

(f)

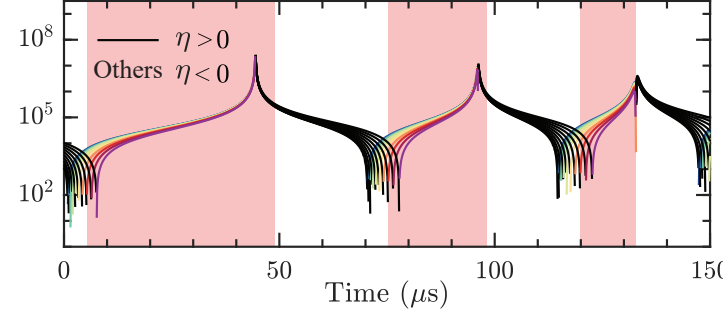

Fig. S8. (a,c,e) Numerically simulated bubble dynamics when $R_{\max }=500 \mu m$. (b,d,f) Calculated bubble wall stretch ratio $\lambda_{w}$ and the variables $\eta$ and $\xi$ calculated using Eqs (7-8) in the main text. Red shaded regions denote theoretically predicted non-spherical mode shape 8 instabilities.
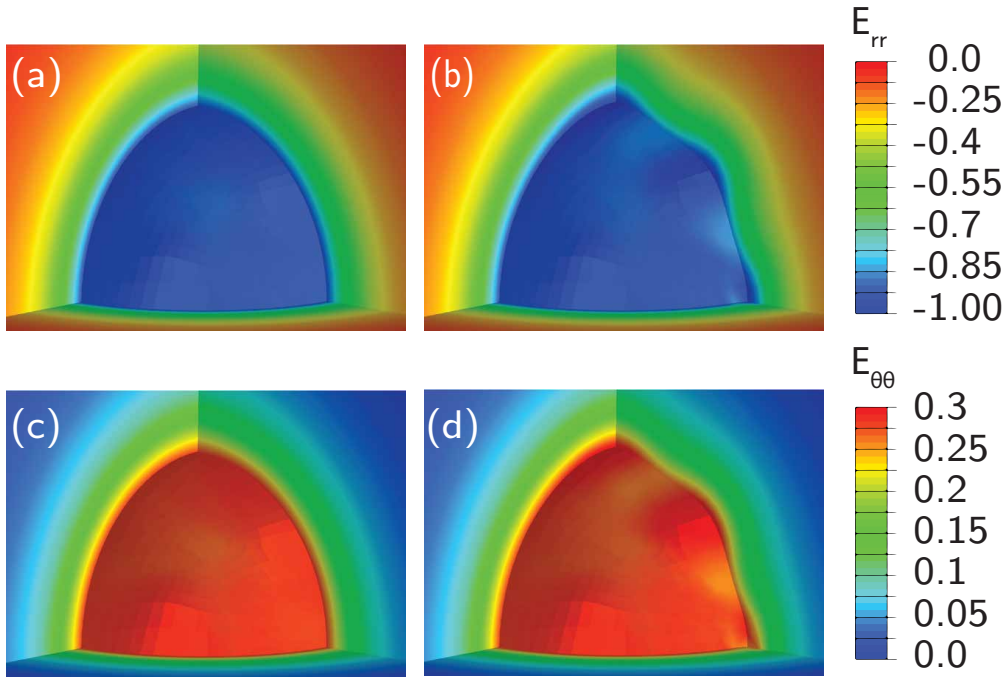

Fig. S9. Contours of the spherical component of the logarithmic strain, $E_{r r}$, for (a) the spherical bubble and (b) the perturbed bubble. Contours of the circumferential component of the logarithmic strain, $E_{\theta \theta}$, for (c) the spherical bubble and (d) the perturbed bubble. The deformed radius of the spherical bubble is $72 \mu \mathrm{m}$, and the perturbation amplitude in (b) and (d) is $1.6386 \mu \mathrm{m}$. 

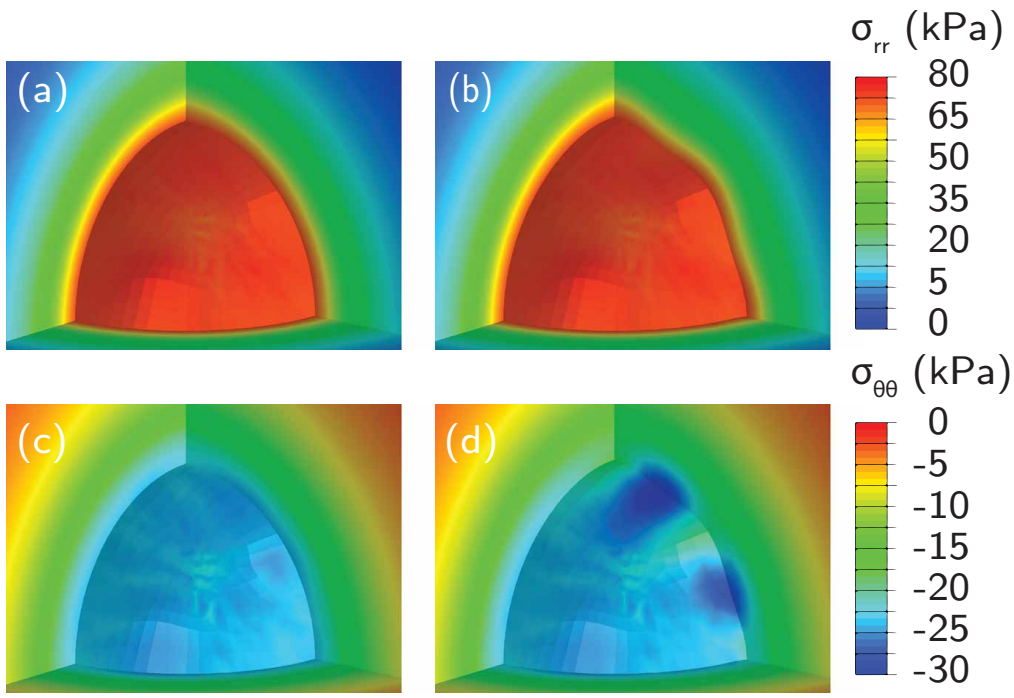

Fig. S10. Contours of the spherical component of the Cauchy stress, $\sigma_{r r}$, for (a) the spherical bubble and (b) the perturbed bubble. Contours of the circumferential component of the Cauchy stress, $\sigma_{\theta \theta}$, for (c) the spherical bubble and (d) the perturbed bubble. The deformed radius of the spherical bubble is $72 \mu \mathrm{m}$, and the perturbation amplitude in (b) and (d) is $1.6386 \mu \mathrm{m}$. 